\title{
University of Washington, Department of Chemistry ${ }^{1}$
}

\begin{tabular}{|c|c|c|c|c|c|}
\hline $\begin{array}{l}\text { Laboratory } \\
\text { number }\end{array}$ & $\begin{array}{l}\text { Published } \\
\text { reference }\end{array}$ & $\begin{array}{l}\text { Original date or } \\
\quad \text { other value }\end{array}$ & $\begin{array}{l}\text { Corrected date } \\
\text { or other value }\end{array}$ & $\partial \mathrm{C}^{14}, \%$ & $\begin{array}{l}\text { A.D./ } \\
\text { B.C. date }\end{array}$ \\
\hline UW-1 & $78: 3$ & $2170 \pm 60$ & & $-245 \pm 5$ & 220 B.C. \\
\hline-2 & $78: 3$ & $780 \pm 60$ & & $-98 \pm 6$ & A.D. 1170 \\
\hline-3 & $78: 7$ & $1690 \pm 80$ & & $-190 \pm 8$ & A.D. 260 \\
\hline-4 & $78: 7$ & $1340 \pm 100$ & & $-154 \pm 10$ & A.D. 610 \\
\hline .5 & $78: 7$ & $1686 \pm 60$ & & $-190 \pm 6$ & A.D. 264 \\
\hline-7 & $78: 4$ & $27,900 \pm 800 \mathrm{~d}$ & $>50,000^{2}$ & & \\
\hline-8 & $78: 4$ & $12,300 \pm 200$ & $10,700 \pm 200 \mathrm{c}$ & $-736 \pm 7$ & 8750 B.C. \\
\hline-9 & $78: 5$ & $31,600 \pm 900$ & $>32,000 \mathrm{c}$ & & \\
\hline-10 & $78: 4$ & $34,700 \pm 1100$ & $>35,000 \mathrm{c}$ & & \\
\hline-11 & $78: 4$ & $31,300 \pm 900$ & $>31,000 \mathrm{c}$ & & \\
\hline-12 & $78: 4$ & $27,900 \pm 1200$ & $>28,000 \mathrm{c}$ & & \\
\hline-13 & $78: 4$ & $31,400 \pm 1600$ & $>31,000 \mathrm{c}$ & & \\
\hline-14 & $78: 6$ & $685 \pm 40$ & & $-88 \pm 4$ & A.D. 1265 \\
\hline-15 & $78: 6$ & $260 \pm 50$ & & $-39 \pm 7$ & A.D. 1690 \\
\hline-16 & $78: 6$ & $1380 \pm 100$ & & $-169 \pm 11$ & A.D. 570 \\
\hline .17 & $78: 4$ & $24,200 \pm 2100 \mathrm{~d}$ & $>49,000^{3}$ & & \\
\hline-18 & $78: 6$ & $3700 \pm 100$ & & & I750 B.C. \\
\hline-19 & $78: 5$ & $33,000 \pm 1000 \mathrm{~d}$ & $>44,000^{4}$ & & \\
\hline-20 & $78: 5$ & $34,500 \pm 1000$ & $>34,000 \mathrm{c}$ & & \\
\hline .21 & $78: 6$ & $433 \pm 80$ & & $-53 \pm 9$ & A.D. 1520 \\
\hline .22 & $78: 7$ & $1715 \pm 60$ & & $-203 \pm 6$ & A.D. 235 \\
\hline-24 & $78: 7$ & $1514 \pm 40$ & & $-181 \pm 4$ & A.D. 436 \\
\hline-25 & $78: 5$ & $32,700 \pm 1000$ & $>33,000 \mathrm{c}$ & & \\
\hline-26 & $78: 7$ & $1540 \pm 70$ & & $-175 \pm 8$ & A.D. 410 \\
\hline .27 & $78: 5$ & $1680 \pm 70$ & & $-188 \pm 8$ & A.D. 270 \\
\hline
\end{tabular}

${ }^{1}$ Reference standard Sequoia wood, not significantly different from 0.95 NBS oxalic acid.

${ }^{2}$ See UW-74, Washington III.

${ }^{3}$ See UW-63, Washington III.

${ }^{4}$ See UW-67, Washington III.

\section{U. S. Geological Survey ${ }^{1}$}

$\begin{array}{ccc}\begin{array}{c}\text { Laboratory } \\ \text { number }\end{array} & \begin{array}{c}\text { Published } \\ \text { reference }\end{array} & \begin{array}{c}\text { Original date or } \\ \text { other value }\end{array} \\ \text { W-7 } & 17: 472 & 2270 \pm 200 \\ -26 & 17: 468 & 2540 \pm 200 \\ -27 & 17: 468 & 2500 \pm 200 \\ -28 & 17: 472 & >30,000 \\ -29 & 17: 467 & 330 \pm 160 \\ -30 & 17: 468 & 4030 \pm 200 \\ -31 & 17: 468 & 300 \pm 160 \\ -32 & 17: 468 & 650 \pm 200 \\ -33 & 17: 469 & 13,600 \pm 500 \\ -35 & 17: 472 & <200 \\ -36 & 17: 472 & 2700 \pm 200 \\ -37 & 17: 469 & 20,700 \pm 600 \\ -40 & 17: 468 & 1050 \pm 160 \\ -42 & 17: 471-2 & 11,350 \pm 120 \\ -43 & 17: 471 & 3500 \pm 200 \\ -44 & 17: 469 & 4800 \pm 400 \\ -45 & 17: 469 & 7570 \pm 250 \\ -46 & 17: 469 & 12,700 \pm 280 \\ -47 & 17: 472 & 980 \pm 150 \\ -48 & 17: 468 & 8500 \pm 200 \\ -49 & 17: 471 & 10,560 \pm 200 \\ -50 & 17: 472 & 4900 \pm 250\end{array}$

Corrected date or other value

$11,250 \pm 120 \mathrm{c}$

$11,250 \pm 120 \mathrm{c}$
A.D./

B.C. date 320 B.C. 590 B.C. 550 B.C.

A.D. 1620 2080 B.C. A.D. 1650 A.D. 1300 11,650 B.C.

750 B.C. 18,750 B.C. A.D. 900 9300 B.C 1550 B.C. 2850 B.C. 5620 B.C. 10,750 B.C. A.D. 970 6550 B.C. 8610 B.C. 2950 B.C. 


\begin{tabular}{|c|c|c|c|c|}
\hline $\begin{array}{l}\text { Laboratory } \\
\text { number }\end{array}$ & $\begin{array}{l}\text { Published } \\
\text { reference }\end{array}$ & $\begin{array}{l}\text { Original date or } \\
\quad \text { other value }\end{array}$ & $\begin{array}{l}\text { Corrected date } \\
\text { or other value }\end{array}$ & $\partial \mathrm{C}^{14}, \%$ \\
\hline-53 & $17: 471$ & $3300 \pm 200$ & & \\
\hline-57 & $17: 469$ & $12,380 \pm 370$ & & \\
\hline-58 & $17: 470$ & $12,380 \pm 360$ & & \\
\hline-59 & $17: 470$ & $7990 \pm 200$ & & \\
\hline-61 & $17: 470$ & $6720 \pm 200$ & & \\
\hline-62 & $17: 471$ & $1100 \pm 200$ & & \\
\hline-64 & $17: 470$ & $13,140 \pm 400$ & & \\
\hline-65 & $17: 470$ & $13,020 \pm 400$ & & \\
\hline-66 & $17: 468$ & $23,000 \pm 800$ & & \\
\hline-67 & $17: 470$ & $>34,000$ & & \\
\hline-68 & $17: 470$ & $22,900 \pm 900$ & & \\
\hline-69 & $17: 470$ & $25,100 \pm 800$ & & \\
\hline-71 & $17: 469$ & $24,600 \pm 800$ & & \\
\hline-72 & $17: 468$ & $8570 \pm 200$ & & \\
\hline-74 & $17: 468$ & $>32,000$ & & \\
\hline-75 & $17: 468$ & $>32,000$ & & \\
\hline-76 & $17: 471$ & $>32,000$ & & \\
\hline-77 & $17: 471$ & $>32,000$ & & \\
\hline-78 & $17: 471$ & $2370 \pm 200$ & & \\
\hline-79 & $17: 470$ & $24,000 \pm 700$ & & \\
\hline-81 & $17: 472$ & $11,170 \pm 180$ & & \\
\hline .82 & $17: 472$ & $10,260 \pm 200$ & & \\
\hline-83 & $17: 471-2$ & $11,410 \pm 180$ & & \\
\hline-84 & $17: 472$ & $10,510 \pm 180$ & & \\
\hline-85 & $17: 472-3$ & $34,000 \pm 2800^{2}$ & & \\
\hline-86 & $17: 472-3$ & $28,500 \pm 800$ & & \\
\hline-87 & $17: 469$ & $>35,000$ & & \\
\hline-88 & $17: 469$ & $21,400 \pm 600$ & & \\
\hline-89 & $17: 472-3$ & $7300 \pm 300$ & & \\
\hline-91 & $17: 469$ & $18,050 \pm 400$ & & \\
\hline-92 & $17: 469$ & $19,980 \pm 500$ & & \\
\hline-95 & $17: 468$ & $8780 \pm 200$ & & \\
\hline-96 & $17: 469$ & $>34,000$ & & \\
\hline-97 & $17: 472-3$ & $12,300 \pm 350$ & & \\
\hline-98 & $17: 472-3$ & $6800 \pm 350$ & & \\
\hline-99 & $17: 470$ & $>31,000$ & & \\
\hline-100 & $17: 471$ & $>32,000$ & & \\
\hline-101 & $17: 471$ & $>32,000$ & & \\
\hline-102 & $17: 471$ & $>36,000$ & & \\
\hline-104 & $17: 472-3$ & $10,600 \pm 400$ & & \\
\hline-112 & $18: 486$ & $>32,000$ & & \\
\hline-115 & $18: 484$ & $>30,000$ & & \\
\hline-121 & $18: 485$ & $>30,000$ & & \\
\hline-125 & $18: 488$ & $3010 \pm 160$ & & \\
\hline-126 & $18: 484$ & $16,720 \pm 600$ & & \\
\hline-127 & $18: 482$ & $21,600 \pm 1000$ & & \\
\hline-130 & $18: 485$ & $<200$ & & \\
\hline-132 & $18: 482$ & $17,200 \pm 500$ & & \\
\hline-133 & $18: 482$ & $>32,000$ & & \\
\hline-134 & $18: 482$ & $15,700 \pm 400$ & & \\
\hline-135 & $18: 482$ & $>33.000$ & & \\
\hline-136 & $18: 485$ & $6380 \pm 350$ & & \\
\hline-139 & $18: 484$ & $>38,000$ & & \\
\hline-140 & $18: 483$ & $12,650 \pm 350$ & & \\
\hline-141 & $18: 484$ & $24,500 \pm 800$ & & \\
\hline-142 & $18: 487$ & $21,500 \pm 700$ & & \\
\hline-143 & $18: 484$ & $2800 \pm 200$ & & \\
\hline-145 & $18: 485$ & $6170 \pm 240$ & & \\
\hline-147 & $18: 482$ & $>33,000$ & & \\
\hline-148 & $18: 482$ & $>32,000$ & & \\
\hline .149 & $18: 486$ & $3300 \pm 240$ & & \\
\hline
\end{tabular}

A.D./

B.C. date

1350 B.C.

10,430 B.C.

10,430 B.C. 6040 B.C.

4770 B.C.

A.D. 850

11,190 B.C.

11,070 B.C.

21,050 B.C.

22,650 B.C.

20,950 B.C.

23,150 B.C.

22,650 B.C.

6620 B.C.

420 B.C.

22,050 B.C.

9220 B.C.

8310 B.C.

9460 B.C.

8560 B.C.

32,050 B.C.

26,550 B.C.

19,450 B.C.

5350 B.C.

16,100 B.C.

18,030 B.C.

6830 B.C.

10,350 B.C.

4850 B.C.

8650 B.C.

1060 B.C.

14,770 B.C.

19,650 B.C.

15,250 B.C.

13,750 B.C.

4430 B.C.

10,700 B.C.

22,550 B.C.

19,550 B.C.

850 B.C.

4220 B.C.

1350 B.C. 


\begin{tabular}{|c|c|c|c|c|}
\hline $\begin{array}{l}\text { Laboratory } \\
\text { number }\end{array}$ & $\begin{array}{l}\text { Published } \\
\text { reference }\end{array}$ & $\begin{array}{l}\text { Original date or } \\
\text { other value }\end{array}$ & $\begin{array}{l}\text { Corrected date } \\
\text { or other value }\end{array}$ & $\partial \mathrm{C}^{14}, \%$ \\
\hline $\begin{array}{l}-150 \\
-151\end{array}$ & $\begin{array}{l}18: 487 \\
18: 487\end{array}$ & $\begin{array}{l}11,750 \pm 600 \\
23,600 \pm 800\end{array}$ & & \\
\hline .152 & $18: 482$ & $>40,000$ & & \\
\hline-153 & $18: 484$ & $14,700 \pm 400$ & & \\
\hline-154 & $18: 487$ & $580 \pm 200$ & & \\
\hline-155 & $18: 487$ & $600 \pm 200$ & & \\
\hline-157 & $18: 485$ & $>38,000$ & & \\
\hline-158 & $18: 482$ & $13,500 \pm 250$ & & \\
\hline-159 & $18: 482$ & $11,800 \pm 300$ & & \\
\hline-160 & $18: 482$ & $3950 \pm 250$ & & \\
\hline-161 & $18: 483$ & $12,200 \pm 350$ & & \\
\hline-162 & $18: 482$ & $27,600 \pm 1000$ & & \\
\hline-164 & $18: 482$ & $21,300 \pm 800$ & & \\
\hline-165 & $18: 483$ & $19,500 \pm 800$ & & \\
\hline-166 & $18: 482$ & $8600 \pm 300$ & & \\
\hline-167 & $18: 482$ & $10,860 \pm 350$ & & \\
\hline-168 & $18: 487$ & $1450 \pm 200$ & & \\
\hline-169 & $18: 488$ & $8500 \pm 250$ & & \\
\hline-170 & $18: 482$ & $4820 \pm 200$ & & \\
\hline-172 & $18: 487$ & $15,750 \pm 800$ & & \\
\hline-173 & $18: 486$ & $>36,000$ & & \\
\hline-174 & $18: 486$ & $>38,000$ & & \\
\hline-175 & 18:486 & $700 \pm 250$ & & \\
\hline-176 & $18: 485$ & $5300 \pm 300$ & & \\
\hline-177 & $18: 485$ & $27,500 \pm 1200$ & & \\
\hline-178 & $18: 488$ & $29,500 \pm 1500$ & & \\
\hline-179 & $18: 488$ & $12,000 \pm 400$ & & \\
\hline-180 & 18:488 & $>34,000$ & & \\
\hline-183 & $18: 486$ & $4020 \pm 200$ & & \\
\hline-184 & 18:482 & $8450 \pm 250$ & & \\
\hline-185 & $18: 482$ & $>30,000$ & & \\
\hline-186 & $18: 483$ & $\geqslant 31,000$ & & \\
\hline-187 & $18: 484$ & $19,200 \pm 700$ & & \\
\hline-188 & $18: 483$ & $23,000 \pm 800$ & & \\
\hline-189 & $18: 485$ & $>40,000$ & & \\
\hline-190 & $18: 485$ & $1100 \pm 250$ & & \\
\hline-191 & $18: 487$ & $24,000 \pm 1000$ & & \\
\hline-192 & $18: 486$ & $>35,000$ & & \\
\hline-194 & $18: 486$ & $>34,000$ & & \\
\hline-195 & $18: 482$ & $>35,000$ & & \\
\hline .196 & $21: 173$ & $>40,000$ & & \\
\hline-197 & $18: 486$ & $>38,000$ & & \\
\hline-198 & $18: 483$ & $14,300 \pm 450$ & & \\
\hline-199 & $18: 483$ & $9640 \pm 250$ & & \\
\hline-200 & $18: 485$ & $1300 \pm 250$ & & \\
\hline-201 & $19: 445$ & $2500 \pm 250$ & & \\
\hline-223 & $19: 446$ & $9700 \pm 600$ & & \\
\hline-224 & $19: 447$ & $33,000 \pm 3000^{2}$ & & \\
\hline-225 & $19: 447$ & $>32,000$ & & \\
\hline-226 & $19: 447$ & $34,000 \pm 3000^{2}$ & & \\
\hline-227 & $19: 443$ & $<300$ & & \\
\hline-228 & $19: 443$ & $6050 \pm 300$ & & \\
\hline-229 & $19: 443$ & $9300 \pm 350$ & & \\
\hline-230 & $19: 446$ & $<200$ & & \\
\hline-231 & $19: 443$ & $12,550 \pm 400$ & & \\
\hline-233 & $19: 443$ & $12,700 \pm 300$ & & \\
\hline-234 & $19: 443$ & $9160 \pm 250$ & & \\
\hline-235 & $19: 446$ & $6800 \pm 300$ & & \\
\hline-236 & $19: 443$ & $3700 \pm 200$ & & \\
\hline-237 & $19: 443$ & $17,500 \pm 500$ & & \\
\hline-238 & $19: 443$ & 36,000 or older & & \\
\hline
\end{tabular}

A.D./

B.C. date

9800 B.C.

21,650 B.C.

12,750 B.C.

A.D. 1370

A.D. 1350

11,550 B.C.

9850 B.C.

2000 B.C.

10,250 B.C.

25,650 B.C.

19,350 B.C.

17,550 B.C.

6650 B.C.

8910 B.C.

A.D. 500

6550 B.C.

2870 B.C.

13,800 B.C.

A.D. 1250

3350 B.C.

25,550 B.C.

27,550 B.C.

10,050 B.C.

2070 B.C.

6500 B.C.

17,250 B.C.

21,050 B.C.

A.D. 850

22,050 B.C.

12,350 B.C.

7690 B.C.

A.D. 650

550 B.C.

7750 B.C.

31,050 B.C.

32,050 B.C.

4100 B.C.

7350 B.C.

10,600 B.C.

10,750 B.C.

7210 B.C.

4850 B.C.

1750 B.C.

15,550 B.C. 


\begin{tabular}{|c|c|c|c|c|}
\hline $\begin{array}{l}\text { Laboratory } \\
\text { number }\end{array}$ & $\begin{array}{l}\text { Published } \\
\text { reference }\end{array}$ & $\begin{array}{l}\text { Original date or } \\
\text { other value }\end{array}$ & $\begin{array}{l}\text { Corrected date } \\
\text { or other value }\end{array}$ & $\partial \mathrm{C}^{14}, \%$ \\
\hline-239 & $19: 443$ & $>38,000$ & & \\
\hline-241 & $19: 445$ & $>37,000$ & $>38,000 \mathrm{c}$ & \\
\hline-242 & $19: 445$ & $>38,000$ & & \\
\hline-243 & $19: 446$ & $>38,000$ & & \\
\hline-244 & $19: 443$ & $>38,000$ & & \\
\hline-245 & $19: 448$ & $5280 \pm 150$ & & \\
\hline-246 & $19: 443$ & $>38,000$ & & \\
\hline-247 & $19: 446$ & $920 \pm 150$ & & \\
\hline-248 & $19: 444$ & $8550 \pm 250$ & & \\
\hline-250 & $19: 445$ & $>32,000$ & & \\
\hline-251 & $19: 445$ & $>32,000$ & & \\
\hline-252 & $19: 443$ & $>35,000$ & & \\
\hline .253 & $19: 443$ & $>35,000$ & & \\
\hline-254 & $19: 446$ & $6410 \pm 160$ & & \\
\hline-255 & $19: 444$ & $12,090 \pm 200$ & & \\
\hline-256 & $19: 444$ & $>37,000$ & & \\
\hline-257 & $19: 444$ & $>37,000$ & & \\
\hline-258 & $19: 444$ & $>37,000$ & & \\
\hline-259 & $19: 444$ & $>37,000$ & & \\
\hline-261 & $19: 447$ & $12,450 \pm 200$ & & \\
\hline-262 & $19: 447$ & $9500 \pm 200$ & & \\
\hline-263 & $19: 444$ & $>37,000$ & & \\
\hline-264 & $19: 447$ & $11,790 \pm 200$ & & \\
\hline-265 & $19: 443$ & $>25,000$ & & \\
\hline-266 & $19: 447$ & $10,100 \pm 250$ & & \\
\hline-267 & $19: 447$ & $8200 \pm 200$ & $8250 \pm 200 \mathrm{c}$ & \\
\hline-268 & $19: 444$ & $<200$ & & \\
\hline-269 & $19: 447$ & $8800 \pm 200$ & $8900 \pm 200 \mathrm{c}$ & \\
\hline-270 & $19: 446$ & $23,150 \pm 500$ & & \\
\hline-271 & $19: 447$ & $11,750 \pm 200$ & & \\
\hline-272 & $19: 446$ & $5780 \pm 160$ & & \\
\hline-273 & $19: 446$ & $5450 \pm 160$ & & \\
\hline .275 & $19: 447$ & $13,400 \pm 800$ & & \\
\hline-276 & $19: 443$ & $27,000 \pm 1500$ & & \\
\hline .277 & $19: 447$ & $>25,000$ & $>24,000 \mathrm{c}$ & \\
\hline-278 & $19: 443$ & $15,300 \pm 300$ & & \\
\hline-279 & $19: 447$ & $24,000 \pm 3000$ & & \\
\hline-280 & $19: 443$ & $2960 \pm 200$ & & \\
\hline-281 & $19: 447$ & $11,870 \pm 200$ & & \\
\hline-283 & 19:448 & $21,000 \pm 500$ & & \\
\hline-284 & $19: 448$ & $3000 \pm 200$ & & \\
\hline-285 & $19: 445$ & $>35,000$ & & \\
\hline-287 & $19: 446$ & $<200$ & & \\
\hline-288 & $19: 446$ & $4885 \pm 160$ & & \\
\hline-289 & $19: 446$ & $1490 \pm 160$ & & \\
\hline-290 & 19:446 & $1360 \pm 200$ & & \\
\hline-291 & $19: 447$ & $3310 \pm 200$ & & \\
\hline-292 & $19: 446$ & $25,000 \pm 800$ & & \\
\hline-294 & $19: 444$ & $>37,000$ & & \\
\hline-295 & $19: 444$ & $>38,000$ & & \\
\hline-297 & $19: 445$ & $4610 \pm 200$ & & \\
\hline-298 & $19: 445$ & $3970 \pm 600$ & & \\
\hline .299 & $19: 445$ & $2800 \pm 180$ & & \\
\hline-300 & $20: 1478$ & $>37,000$ & & \\
\hline-301 & $20: 1478$ & $>37,000$ & & \\
\hline-302 & $20: 1484$ & $>38,000$ & & \\
\hline-303 & $20: 1486$ & $>38,000$ & & \\
\hline-304 & $20: 1477$ & $20,500 \pm 800$ & & \\
\hline-305 & $20: 1486$ & $>38,000$ & & \\
\hline .306 & $20: 1482$ & $975 \pm 160$ & & \\
\hline-307 & $20: 1480$ & $>37,000$ & & \\
\hline
\end{tabular}

A.D./

B.C. date

3330 B.C.

A.D. 1030

6600 B.C.

4460 B.C.

10,140 B.C.

10,500 B.C.

7550 B.C.

9840 B.C.

8150 B.C.

6300 B.C.

6950 B.C.

21,200 B.C.

9800 B.C.

3830 B.C.

3500 B.C.

11,450 B.C.

25,050 B.C.

13,350 B.C.

22,050 B.C.

1010 B.C.

9920 B.C.

19,050 B.C.

1050 B.C.

2935 B.C.

A.D. 460

A.D. 590

1360 B.C.

23,050 B.C.

2660 B.C.

2020 B.C.

850 B.C.

18,550 B.C.

A.D. 975 


\begin{tabular}{|c|c|c|c|}
\hline $\begin{array}{l}\text { Laboratory } \\
\text { number }\end{array}$ & $\begin{array}{l}\text { Published } \\
\text { reference }\end{array}$ & $\begin{array}{l}\text { Original date or } \\
\text { other value }\end{array}$ & $\begin{array}{l}\text { Corrected date } \\
\text { or other value }\end{array}$ \\
\hline-308 & $20: 1484$ & $>34,000$ & \\
\hline-310 & $20: 1486$ & $<200$ & \\
\hline .311 & $20: 1485$ & $730 \pm 200$ & \\
\hline-312 & $20: 1478$ & $27,000 \pm 800$ & $27,100 \pm 800 \mathrm{c}$ \\
\hline-313 & $20: 1476$ & $650 \pm 60$ & \\
\hline-314 & $20: 1476$ & $840 \pm 60$ & \\
\hline-315 & $20: 1483$ & $>37,000$ & \\
\hline-316 & $20: 1484$ & $5520 \pm 160$ & \\
\hline-317 & $20: 1477$ & $8340 \pm 250$ & \\
\hline-318 & $20: 1480$ & $1385 \pm 200$ & \\
\hline-319 & $20: 1484$ & $16,680 \pm 300$ & \\
\hline-321 & $20: 1484$ & $16,850 \pm 300$ & \\
\hline .322 & $20: 1486$ & $3230 \pm 160$ & \\
\hline-323 & $20: 1483$ & $26,480 \pm 800$ & \\
\hline-325 & $20: 1477$ & $9755 \pm 300$ & \\
\hline .326 & $20: 1484$ & $9925 \pm 300$ & \\
\hline-327 & $20: 1476$ & $16,955 \pm 400$ & \\
\hline-328 & $20: 1477$ & $390 \pm 160$ & \\
\hline-329 & $20: 1477$ & $<200$ & \\
\hline-330 & $20: 1476$ & $1675 \pm 200$ & \\
\hline-331 & $20: 1477$ & $18,000 \pm 400$ & \\
\hline-333 & $20: 1478$ & $25,700 \pm 800$ & \\
\hline-334 & $20: 1478$ & $22,450 \pm 1000$ & \\
\hline-335 & $20: 1484$ & $8330 \pm 300$ & \\
\hline-336 & $20: 1483$ & $9870 \pm 250$ & \\
\hline-337 & $20: 1486$ & $1710 \pm 160$ & \\
\hline-339 & $20: 1479$ & $>38,000$ & \\
\hline-340 & $20: 1484$ & $26,700 \pm 2000$ & \\
\hline-341 & $20: 1484$ & $23,000 \pm 1400$ & \\
\hline-342 & $20: 1486$ & $1610 \pm 160$ & \\
\hline-343 & $20: 1484$ & $29,500 \pm 2000$ & \\
\hline-344 & $20: 1483$ & $>38,000$ & \\
\hline-345 & $20: 1483$ & $9130 \pm 250$ & \\
\hline-346 & $20: 1477$ & $13,000 \pm 300$ & \\
\hline-347 & $20: 1477$ & $9430 \pm 300$ & \\
\hline-349 & $20: 1478$ & $20,340 \pm 750$ & \\
\hline .350 & $20: 1485$ & $+5.4 \%$ & $105.4 \%$ of modern \\
\hline-351 & $20: 1476$ & $635 \pm 60$ & \\
\hline-352 & $20: 1476$ & $630 \pm 60$ & \\
\hline-353 & $20: 1483$ & $8250 \pm 200$ & \\
\hline-354 & $20: 1478$ & $12,030 \pm 200$ & \\
\hline-356 & $20: 1477$ & $22,480 \pm 800$ & \\
\hline-357 & $20: 1483$ & $>35,000$ & \\
\hline-358 & $20: 1485$ & $>32,000$ & \\
\hline-359 & $20: 1486$ & $<200$ & \\
\hline-360 & $20: 1483$ & $11,930 \pm 250$ & \\
\hline-361 & $20: 1477$ & $10,800 \pm 250$ & \\
\hline-362 & $20: 1485$ & $>33,000$ & \\
\hline-363 & $20: 1484$ & $4320 \pm 250$ & \\
\hline-365 & $20: 1477$ & $14,000 \pm 350$ & \\
\hline-367 & $20: 1483$ & $9200 \pm 250$ & \\
\hline-368 & $20: 1480$ & $>33,000$ & \\
\hline-369 & $20: 1482$ & $1050 \pm 160$ & \\
\hline-370 & $20: 1477$ & $3400 \pm 250$ & \\
\hline-371 & $20: 1482$ & $390 \pm 160$ & \\
\hline-372 & $20: 1486$ & $3950 \pm 200$ & \\
\hline-373 & $20: 1481$ & $>35,000$ & \\
\hline-374 & $20: 1482$ & $1200 \pm 160$ & \\
\hline-376 & $20: 1482$ & $390 \pm 160$ & \\
\hline-377 & $20: 1482$ & $>35,000$ & \\
\hline-378 & $20: 1482$ & $2500 \pm 200$ & \\
\hline
\end{tabular}

A.D./

B.C. date

A.D. 1220

25,150 B.C.

A.D. 1300

A.D. 1110

3570 B.C.

6390 B.C.

A.D. 565

14,730 B.C.

14,900 B.C.

1280 B.C.

24,530 B.C.

7805 B.C.

7975 B.C.

15,005 B.C.

A.D. 1560

A.D. 275

16,050 B.C.

23,750 B.C.

20,500 B.C.

6380 B.C.

7920 B.C.

A.D. 240

24,750 B.C.

21,050 B.C.

A.D. 340

27,550 B.C.

7180 B.C.

11,050 B.C.

7480 B.C.

18,390 B.C.

A.D. 1315

A.D. 1320

6300 B.C.

10,080 B.C.

20,530 B.C.

9980 B.C.

8850 B.C.

2370 B.C.

12,050 B.C. 7250 B.C.

A.D. 900

1450 B.C.

A.D. 1560

2000 B.C.

A.D. 750

A.D. 1550

550 B.C. 


\begin{tabular}{|c|c|c|c|c|}
\hline $\begin{array}{l}\text { Laboratory } \\
\text { number }\end{array}$ & $\begin{array}{l}\text { Published } \\
\text { reference }\end{array}$ & $\begin{array}{l}\text { Original date or } \\
\text { other value }\end{array}$ & $\begin{array}{l}\text { Corrected date } \\
\text { or other value }\end{array}$ & $\partial \mathrm{C}^{14}, \% 0$ \\
\hline-379 & $20: 1478$ & $24,700 \pm 1000$ & & \\
\hline-380 & $20: 1481$ & $>38,000$ & & \\
\hline-381 & $20: 1478$ & $15,600 \pm 600$ & & \\
\hline-382 & $20: 1484$ & $12,960 \pm 350$ & & \\
\hline-383 & $20: 1476$ & $21,300 \pm 1000$ & & \\
\hline-384 & $20: 1476$ & $19,300 \pm 1000$ & & \\
\hline-385 & $20: 1484$ & $7720 \pm 300$ & & \\
\hline-386 & $20: 1484$ & $9730 \pm 350$ & & \\
\hline-388 & $20: 1478$ & $9930 \pm 280$ & & \\
\hline-389 & $20: 1478$ & $12,700 \pm 250$ & & \\
\hline-390 & $20: 1477$ & $1730 \pm 160$ & & \\
\hline-391 & $20: 1479$ & $>38,000$ & & \\
\hline-392 & $20: 1479$ & $9580 \pm 250$ & & \\
\hline-393 & $20: 1479$ & $8800 \pm 250$ & & \\
\hline-394 & $20: 1479$ & $11,500 \pm 300$ & & \\
\hline-395 & $20: 1476$ & $200 \pm 160$ & & \\
\hline-396 & $20: 1476$ & $2980 \pm 180$ & & \\
\hline-397 & $20: 1479$ & $9230 \pm 320$ & & \\
\hline-398 & $20: 1479$ & $12,900 \pm 330$ & & \\
\hline-399 & $20: 1478$ & $20,700 \pm 650$ & & \\
\hline-4.01 & $20: 1485$ & $10,200 \pm 350$ & & \\
\hline .402 & $20: 1478$ & $11,220 \pm 300$ & & \\
\hline-405 & $20: 1482$ & $3250 \pm 200$ & & \\
\hline-406 & $20: 1478$ & $26,150 \pm 700$ & & \\
\hline-407 & $20: 1486$ & $<200$ & & \\
\hline-408 & $20: 1483$ & $4760 \pm 220$ & & \\
\hline .409 & $20: 1484$ & $11,300 \pm 300$ & & \\
\hline-410 & $20: 1484$ & $>32,000$ & & \\
\hline-411 & $20: 1484$ & $14,000 \pm 450$ & & \\
\hline-412 & $20: 1484$ & $14,000 \pm 450$ & & \\
\hline-414 & $20: 1477$ & $22,000 \pm 1000$ & & \\
\hline-415 & $20: 1477$ & $>37,000$ & & \\
\hline-416 & $20: 1479$ & $12,900 \pm 300$ & & \\
\hline-4.17 & $20: 1486$ & $>38,000$ & $>35,000 \mathrm{c}$ & \\
\hline-4.18 & $20: 1484$ & $9400 \pm 250$ & & \\
\hline-419 & $20: 1486$ & $1100 \pm 160$ & & \\
\hline-420 & $20: 1480$ & $>38,000$ & & \\
\hline$-4,21$ & $20: 1485$ & $470 \pm 160$ & & \\
\hline$-4,22$ & $20: 1486$ & $2080 \pm 160$ & & \\
\hline-423 & $20: 1486$ & $>38,000$ & & \\
\hline-424 & $20: 1486$ & $<200$ & & \\
\hline-4.25 & $20: 1478$ & $5370 \pm 200$ & & \\
\hline-426 & $20: 1478$ & $10,700 \pm 300$ & & \\
\hline-428 & $20: 1484$ & $4030 \pm 160$ & & \\
\hline-429 & $20: 1482$ & $11,400 \pm 400$ & & \\
\hline-430 & $20: 1477$ & $12,920 \pm 400$ & & \\
\hline-431 & $20: 1481$ & $8000 \pm 300$ & & \\
\hline-432 & $20: 1481$ & $3540 \pm 300$ & & \\
\hline-433 & $20: 1481$ & $480 \pm 160$ & & \\
\hline-434 & $20: 1479$ & $6040 \pm 240$ & & \\
\hline-435 & $20: 1479$ & $23,900 \pm 1000$ & & \\
\hline-436 & $20: 1485$ & $2240 \pm 250$ & & \\
\hline-437 & $20: 1486$ & $2800 \pm 160$ & & \\
\hline-439 & $20: 1484$ & $11,420 \pm 300$ & & \\
\hline-440 & $20: 1484$ & $10,260 \pm 300$ & & \\
\hline-442 & $20: 1484$ & $12,050 \pm 400$ & & \\
\hline-443 & $20: 1477$ & $3710 \pm 250$ & & \\
\hline-4.44 & $20: 1483$ & $>32,000$ & & \\
\hline-445 & $20: 1478$ & $10.200 \pm 300$ & & \\
\hline .446 & $20: 1476$ & $17,000 \pm 800$ & & \\
\hline-447 & $20: 1476$ & $2490 \pm 400$ & & \\
\hline
\end{tabular}

A.D./

B.C. date

22,750 B.C.

13,650 B.C.

11,010 B.C.

19,350 B.C.

17,350 B.C.

5770 B.C.

7780 B.C.

7980 B.C.

10,750 B.C.

A.D. 220

7630 B.C.

6850 B.C.

9550 B.C.

A.D. 1750

1030 B.C.

7280 B.C.

10,950 B.C.

18,750 B.C.

8250 B.C.

9270 B.C.

1300 B.C.

24,200 B.C.

2810 B.C.

9350 B.C.

12,050 B.C.

12,050 B.C.

20,050 B.C.

10,950 B.C.

7450 B.C.

A.D. 850

A.D. 1480

130 B.C.

3420 B.C.

8750 B.C.

2080 B.C.

9450 B.C.

10,970 B.C.

6050 B.C.

1590 B.C.

A.D. 1470

4090 B.C.

21,950 B.C.

290 B.C.

850 B.C.

9470 B.C.

8310 B.C.

10,100 B.C.

1760 B.C.

8250 B.C.

15,050 B.C.

540 B.C. 


\begin{tabular}{|c|c|c|c|c|}
\hline $\begin{array}{l}\text { Laboratory } \\
\text { number }\end{array}$ & $\begin{array}{l}\text { Published } \\
\text { reference }\end{array}$ & $\begin{array}{l}\text { Original date or } \\
\text { other value }\end{array}$ & $\begin{array}{l}\text { Corrected date } \\
\text { or other value }\end{array}$ & $\partial \mathrm{C}^{14}, \%$ \\
\hline-448 & $20: 1476$ & $450 \pm 200$ & & \\
\hline-449 & $20: 1476$ & $12,400 \pm 600$ & & \\
\hline-450 & $20: 1476$ & $15,600 \pm 800$ & & \\
\hline-451 & $20: 1476$ & $15,440 \pm 800$ & & \\
\hline-452 & $20: 1476$ & $16,730 \pm 850$ & & \\
\hline-453 & $20: 1476$ & $1025 \pm 400$ & & \\
\hline-454 & $20: 1478$ & $11,790 \pm 200$ & & \\
\hline .455 & $20: 1484$ & $14,030 \pm 500$ & & \\
\hline-456 & $20: 1484$ & $11,650 \pm 450$ & & \\
\hline-458 & $20: 1484$ & $14,380 \pm 500$ & & \\
\hline-459 & $20: 1485$ & $7570 \pm 400$ & & \\
\hline-461 & $20: 1480$ & $10,050 \pm 270$ & & \\
\hline-462 & $20: 1483$ & $5120 \pm 220$ & & \\
\hline-463 & $20: 1480$ & $13,040 \pm 300$ & & \\
\hline-465 & $20: 1478$ & $4890 \pm 200$ & & \\
\hline-466 & $20: 1478$ & $11,830 \pm 200$ & & \\
\hline-467 & $20: 1478$ & $<200$ & & \\
\hline-468 & $20: 1478$ & $>36,000$ & & \\
\hline-469 & $20: 1478$ & $17,150 \pm 600$ & & \\
\hline-470 & $20: 1478$ & $17,800 \pm 600$ & & \\
\hline-472 & $20: 1479$ & $8140 \pm 300$ & & \\
\hline-473 & $20: 1480$ & $2470 \pm 250$ & & \\
\hline-474 & $20: 1479$ & $10,370 \pm 350$ & & \\
\hline-475 & $20: 1479$ & $>35,000$ & & \\
\hline-476 & $20: 1479$ & $>35,000$ & & \\
\hline-477 & $20: 1486$ & $2000 \pm 250$ & & \\
\hline-478 & $20: 1486$ & $2070 \pm 250$ & & \\
\hline-479 & $20: 1486$ & $4050 \pm 200$ & & \\
\hline-480 & $20: 1486$ & $3950 \pm 200$ & & \\
\hline-482 & $20: 1486$ & $8065 \pm 400$ & & \\
\hline-483 & $20: 1478$ & $20,500 \pm 600$ & & \\
\hline-484 & $20: 1480$ & $2770 \pm 300$ & & \\
\hline-485 & $20: 1480$ & $9690 \pm 400$ & & \\
\hline-486 & $20: 1486$ & $6120 \pm 300$ & & \\
\hline-487 & $20: 1482$ & $9240 \pm 300$ & & \\
\hline-488 & $20: 1486$ & $8540 \pm 350$ & & \\
\hline-490 & $20: 1484$ & $16,530 \pm 800$ & & \\
\hline-491 & $20: 1484$ & $13,380 \pm 400$ & & \\
\hline-493 & $20: 1486$ & $7940 \pm 300$ & & \\
\hline-494 & $20: 1484$ & $18,000 \pm 1000$ & & \\
\hline-495 & $20: 1485$ & $12,900 \pm 350$ & & \\
\hline-497 & $20: 1478$ & $1900 \pm 250$ & & \\
\hline-498 & $20: 1485$ & $11,850 \pm 350$ & & \\
\hline-4.99 & $20: 1485$ & $12,270 \pm 450$ & & \\
\hline-500 & $21: 148$ & $>35,000$ & & \\
\hline-501 & $21: 148$ & $>35,000$ & & \\
\hline-502 & $21: 148$ & $11,710 \pm 325$ & & \\
\hline-503 & $21: 145$ & $>29,000$ & & \\
\hline-504 & $21: 133$ & $>35,000$ & & \\
\hline-506 & $21: 134$ & $1400 \pm 250$ & & \\
\hline-507 & $21: 134$ & $12,000 \pm 300$ & & \\
\hline-508 & $21: 129$ & $2810 \pm 200$ & & \\
\hline-509 & $21: 130$ & $1280 \pm 200$ & & \\
\hline-510 & $21: 130$ & $3250 \pm 200$ & & \\
\hline-512 & $21: 143$ & $14,470 \pm 400$ & & \\
\hline-513 & $21: 143$ & $13,820 \pm 400$ & & \\
\hline-514 & $21: 143$ & $>35,000$ & & \\
\hline-516 & $21: 146$ & $>35,000$ & & \\
\hline-517 & $21: 143$ & $13,910 \pm 400$ & & \\
\hline-518 & $21: 129$ & $>35,000$ & & \\
\hline-519 & $21: 129$ & $>35,000$ & & \\
\hline
\end{tabular}

A.D./

B.C. date

A.D. 1500

10,450 B.C.

13,650 B.C.

13,490 B.C.

14,780 B.C.

A.D. 925

9840 B.C.

12,080 B.C.

9700 B.C.

12,430 B.C.

5620 B.C.

8100 B.C.

3170 B.C.

11,090 B.C.

2940 B.C.

9880 B.C.

15,200 B.C.

15,850 B.C.

6190 B.C.

520 B.C.

8420 B.C.

50 B.C.

120 B.C.

2100 B.C.

2000 B.C.

6115 B.C.

18,550 B.C.

820 B.C.

7740 B.C.

4170 B.C.

7290 B.C.

6590 B.C.

14,580 B.C.

11,430 B.C.

5990 B.C.

16,050 B.C.

10,950 B.C.

A.D. 50

9900 B.C.

10,320 B.C.

9760 B.C.

A.D. 550

10,050 B.C

860 B.C.

A.D. 670

1300 B.C.

12,520 B.C.

11,870 B.C.

11,950 B.C. 


\begin{tabular}{|c|c|c|c|c|}
\hline $\begin{array}{l}\text { Laboratory } \\
\text { number }\end{array}$ & $\begin{array}{c}\text { Published } \\
\text { reference }\end{array}$ & $\begin{array}{l}\text { Original date or } \\
\text { other value }\end{array}$ & $\begin{array}{l}\text { Corrected date } \\
\text { or other value }\end{array}$ & $\partial \mathrm{C}^{14}, \% 0$ \\
\hline-520 & $21: 147$ & $18,530 \pm 500$ & & \\
\hline .521 & $21: 147$ & $<200$ & & \\
\hline-522 & $21: 130$ & $>35,000$ & & \\
\hline .523 & $21: 174$ & $<200$ & & \\
\hline .524 & $21: 136$ & $18,460 \pm 500$ & & \\
\hline-525 & $21: 176$ & $10,800 \pm 300$ & & \\
\hline .526 & $21: 136$ & $29,000 \pm 1200$ & & \\
\hline-528 & $21: 176$ & $11,150 \pm 200$ & & \\
\hline .529 & $21: 162$ & $>35,000$ & & \\
\hline .531 & $21: 170$ & $>38,000$ & & \\
\hline-532 & $21: 176$ & $<200$ & & \\
\hline-534 & $21: 145$ & $>34,000$ & & \\
\hline-535 & $21: 164$ & $>38,000$ & & \\
\hline-536 & $21: 165$ & $9300 \pm 250$ & & \\
\hline-537 & $21: 177$ & $520 \pm 200$ & & \\
\hline-538 & $21: 161$ & $13,050 \pm 400$ & & \\
\hline .540 & $21: 165$ & $11,600 \pm 300$ & & \\
\hline .541 & $21: 165$ & $620 \pm 200$ & & \\
\hline-542 & $21: 152$ & $11,480 \pm 300$ & & \\
\hline-543 & $21: 180$ & $2720 \pm 220$ & & \\
\hline-544 & $21: 130$ & $21,200 \pm 1000$ & & \\
\hline-545 & $21: 180$ & $3680 \pm 250$ & & \\
\hline .546 & $21: 174$ & $3200 \pm 200$ & & \\
\hline .547 & $21: 174$ & $<200$ & & \\
\hline-548 & $21: 144$ & $11,660 \pm 250$ & & \\
\hline .549 & $21: 143$ & $8170 \pm 200$ & & \\
\hline .551 & $21: 143$ & $6570 \pm 200$ & & \\
\hline-552 & $21: 144$ & $11,790 \pm 250$ & & \\
\hline-553 & $21: 144$ & $8110 \pm 200$ & & \\
\hline .554 & $21: 143$ & $6580 \pm 200$ & $6580 \pm 250 \mathrm{c}$ & \\
\hline .555 & $21: 177$ & $5370 \pm 200$ & & \\
\hline-556 & $21: 147$ & $9040 \pm 300$ & & \\
\hline .557 & $21: 144$ & $10,050 \pm 250$ & & \\
\hline-558 & $21: 162$ & $>35,000$ & & \\
\hline-559 & $21: 169$ & $830 \pm 160$ & & \\
\hline-560 & $21: 148$ & $11,540 \pm 200$ & & \\
\hline-562 & $21: 148$ & $4360 \pm 160$ & & \\
\hline .563 & $21: 134$ & $>35,000$ & & \\
\hline .564 & $21: 163$ & $4700 \pm 250$ & & \\
\hline .565 & $21: 163$ & $530 \pm 200$ & & \\
\hline .566 & $21: 163$ & $2170 \pm 200$ & & \\
\hline .568 & $21: 165$ & $9000 \pm 400$ & & \\
\hline-569 & $21: 166$ & $2030 \pm 400$ & & \\
\hline-570 & $21: 130$ & $2130 \pm 200$ & & \\
\hline-571 & $21: 133$ & $2880 \pm 200$ & & \\
\hline-573 & $21: 168$ & $3620 \pm 250$ & & \\
\hline-574 & $21: 148$ & $10,620 \pm 400$ & & \\
\hline .577 & $21: 139$ & $20,500 \pm 800$ & & \\
\hline-578 & $21: 139$ & $>37,000$ & & \\
\hline .579 & $21: 140$ & $20,800 \pm 800$ & & \\
\hline-580 & $21: 140$ & $20,900 \pm 800$ & & \\
\hline-581 & $21: 155$ & $10,540 \pm 350$ & & \\
\hline .582 & $21: 131$ & $1640 \pm 240$ & & \\
\hline .583 & $21: 168$ & $13,280 \pm 400$ & & \\
\hline-584 & $21: 131$ & $2040 \pm 240$ & & \\
\hline-586 & $21: 131$ & $5500 \pm 300$ & & \\
\hline-587 & $21: 131$ & $<200$ & & \\
\hline .588 & $21: 178$ & $8500 \pm 500$ & & \\
\hline-589 & $21: 130$ & $<200$ & & \\
\hline-590 & $21: 153$ & $11,140 \pm 300$ & & \\
\hline-591 & $21: 146$ & $>37,000$ & & \\
\hline
\end{tabular}

A.D./

B.C. date

16,580 B.C.

16,510 B.C. 8850 B.C.

27,050 B.C. 9200 B.C.

7350 B.C

A.D. 1430

11,100 B.C. 9650 B.C.

A.D. 1330

9530 B.C.

770 B.C.

19,250 B.C.

1730 B.C.

1250 B.C.

9710 B.C.

6220 B.C.

4620 B.C.

9810 B.C.

6160 B.C.

4630 B.C.

3420 B.C.

7090 B.C.

8100 B.C.

A.D. 1120

9590 B.C.

2410 B.C.

2750 B.C.

A.D. 1420

220 B.C.

7050 B.C.

80 B.C.

180 B.C.

930 B.C.

1670 B.C.

8670 B.C.

18,550 B.C.

18,850 B.C.

18,950 B.C. 8590 B.C.

A.D. 310

11,330 B.C. 90 B.C.

3550 B.C.

6550 B.C.

9190 B.C. 


\begin{tabular}{|c|c|c|c|c|}
\hline $\begin{array}{l}\text { Laboratory } \\
\text { number }\end{array}$ & $\begin{array}{l}\text { Published } \\
\text { reference }\end{array}$ & $\begin{array}{l}\text { Original date or } \\
\text { other value }\end{array}$ & $\begin{array}{l}\text { Corrected date } \\
\text { or other value }\end{array}$ & $\partial \mathrm{C}^{14}, \% 0$ \\
\hline-592 & $21: 171$ & $850 \pm 200$ & & \\
\hline-593 & $21: 170$ & $1900 \pm 200$ & & \\
\hline-594 & $21: 158$ & $8800 \pm 300$ & & \\
\hline-595 & $21: 140$ & $22,300 \pm 800$ & & \\
\hline-597 & $21: 140$ & $20,300 \pm 800$ & & \\
\hline-598 & $21: 140$ & $20,100 \pm 800$ & & \\
\hline-599 & $21: 146$ & $>37,000$ & & \\
\hline-600 & $21: 146$ & $>37,000$ & & \\
\hline-601 & $21: 161$ & $<250$ & & \\
\hline-602 & $21: 169$ & $8420 \pm 300$ & & \\
\hline-603 & $21: 169$ & $8640 \pm 280$ & & \\
\hline-607 & $21: 182$ & $9040 \pm 250$ & & \\
\hline-608 & $21: 182$ & $7750 \pm 250$ & & \\
\hline-609 & $21: 182$ & $3030 \pm 200$ & & \\
\hline-610 & $21: 134$ & $>38,000$ & & \\
\hline-611 & $21: 135$ & $>38,000$ & & \\
\hline-612 & $21: 134$ & $>38,000$ & & \\
\hline-613 & $21: 134$ & $>38,000$ & & \\
\hline-614 & $21: 151$ & $2800 \pm 200$ & & \\
\hline-616 & $21: 156$ & $1050 \pm 250$ & & \\
\hline-617 & $21: 183$ & $7950 \pm 250$ & & \\
\hline-618 & $21: 151$ & $22.200 \pm 1000$ & & \\
\hline-619 & $21: 177$ & $3840 \pm 300$ & & \\
\hline-620 & $21: 177$ & $2700 \pm 250$ & & \\
\hline-621 & $21: 154$ & $3100 \pm 250$ & & \\
\hline-622 & $21: 155$ & $1040 \pm 250$ & & \\
\hline-623 & $21: 183$ & $7570 \pm 250$ & & \\
\hline-625 & $21: 144$ & $13,030 \pm 250$ & & \\
\hline-626 & $21: 144$ & $12,970 \pm 250$ & & \\
\hline-627 & $21: 183$ & $6550 \pm 200$ & & \\
\hline-628 & $21: 160$ & $>38,000$ & & \\
\hline-629 & $21: 155$ & $700 \pm 200$ & & \\
\hline-630 & $21: 181$ & $4050 \pm 200$ & & \\
\hline-631 & $21: 181$ & $4450 \pm 200$ & & \\
\hline-632 & $21: 181$ & $4190 \pm 200$ & & \\
\hline-634 & $21: 148$ & $>32,000$ & & \\
\hline-635 & $21: 149$ & $12,090 \pm 300$ & & \\
\hline-636 & $21: 180$ & $8280 \pm 1000$ & & \\
\hline-637 & $21: 131$ & $190 \pm 150$ & & \\
\hline-638 & $21: 153$ & $31,800 \pm 1200$ & & \\
\hline-639 & $21: 131$ & $500 \pm 150$ & & \\
\hline-640 & $21: 143$ & $>36,000$ & & \\
\hline-641 & $21: 153$ & $12,000 \pm 500$ & & \\
\hline-642 & $21: 137$ & $26,200 \pm 800$ & & \\
\hline-643 & $21: 178$ & $>38,000$ & & \\
\hline-644 & $21: 169$ & $>40,000$ & & \\
\hline-645 & $21: 141$ & $19,940 \pm 300$ & & \\
\hline-646 & $21: 161$ & $>38,000$ & & \\
\hline-647 & $21: 131$ & $>38,000$ & & \\
\hline-648 & $21: 152$ & $21,600 \pm 400$ & & \\
\hline-650 & $21: 184$ & $33,300 \pm 1000$ & & \\
\hline-651 & $21: 182$ & $8830 \pm 200$ & & \\
\hline-652 & $21: 182$ & $7950 \pm 200$ & & \\
\hline-654 & $21: 184$ & $28,700 \pm 700$ & & \\
\hline .655 & $21: 184$ & $1380 \pm 300$ & & \\
\hline-656 & $21: 184$ & $3420 \pm 200$ & & \\
\hline-657 & $21: 182$ & $11,240 \pm 300$ & & \\
\hline-658 & $21: 175$ & $3760 \pm 160$ & & \\
\hline-659 & $21: 175$ & $3480 \pm 160$ & & \\
\hline-660 & $21: 183$ & $7040 \pm 200$ & & \\
\hline-661 & $21: 161$ & $<250$ & & \\
\hline
\end{tabular}

A.D./

B.C. date

A.D. 1100

A.D. 50

6850 B.C.

20,350 B.C.

18,350 B.C.

18,150 B.C.

6470 B.C.

6690 B.C.

7090 B.C.

5800 B.C.

1080 B.C.

850 B.C.

A.D. 900

6000 B.C.

20,250 B.C.

1890 B.C.

750 B.C.

1150 B.C.

A.D. 910

5620 B.C.

11,080 B.C.

11,020 B.C.

4600 B.C.

A.D. 1250

2100 B.C.

2500 B.C.

2240 B.C.

10,140 B.C.

6330 B.C.

A.D. 1760

29,850 B.C.

A.D. 1450

10,050 B.C.

24,250 B.C.

17,990 B.C.

19,650 B.C.

31,350 B.C.

6880 B.C.

6000 B.C.

26,750 B.C.

A.D. 570

1470 B.C

9290 B.C.

1810 B.C.

1530 B.C.

5090 B.C. 


\begin{tabular}{|c|c|c|c|c|c|}
\hline $\begin{array}{l}\text { Laboratory } \\
\text { number }\end{array}$ & $\begin{array}{l}\text { Published } \\
\text { reference }\end{array}$ & $\begin{array}{l}\text { Original date or } \\
\text { other value }\end{array}$ & $\begin{array}{l}\text { Corrected date } \\
\text { or other value }\end{array}$ & $\partial \mathrm{C}^{14}, \%$ & $\begin{array}{l}\text { A.D./ } \\
\text { B.C. date }\end{array}$ \\
\hline-662 & $21: 183$ & $<200$ & & & \\
\hline-663 & $21: 141$ & $23,300 \pm 600$ & & & 21,350 B.C. \\
\hline-665 & $21: 182$ & $11,200 \pm 200$ & & & 9250 B.C. \\
\hline-666 & $21: 141$ & $4040 \pm 250$ & & & 2090 B.C. \\
\hline-667 & $21: 183$ & $10,600 \pm 300$ & & & 8650 B.C. \\
\hline-668 & $21: 141$ & $21,400 \pm 650$ & & & 19,450 B.C. \\
\hline-669 & $21: 141$ & $>36,000$ & & & \\
\hline-670 & $21: 154$ & $12,200 \pm 400$ & & & 10,250 B.C. \\
\hline-671 & $21: 163$ & $>38,000$ & & & \\
\hline-672 & $21: 163$ & $>38,000$ & & & \\
\hline-674 & $21: 166$ & $2000 \pm 200$ & & & 50 B.C. \\
\hline-675 & $21: 132$ & $770 \pm 100$ & & & A.D. 1180 \\
\hline-676 & $21: 132$ & $5480 \pm 120$ & & & 3530 B.C. \\
\hline-677 & $21: 132$ & $400 \pm 100$ & & & A.D. 1550 \\
\hline-678 & $21: 132$ & $1880 \pm 100$ & & & A.D. 70 \\
\hline-680 & $21: 142$ & $640 \pm 200$ & & & A.D. 1310 \\
\hline-681 & $21: 184$ & $10,800 \pm 300$ & $10,870 \pm 300 \mathrm{c}$ & & 8920 B.C. \\
\hline-693 & $21: 147$ & $9500 \pm 600$ & & & 7550 B.C. \\
\hline-695 & $21: 132$ & $7630 \pm 200$ & & & 5680 B.C. \\
\hline-696 & $21: 132$ & $9650 \pm 250$ & & & 7700 B.C. \\
\hline-697 & $21: 132$ & $9870 \pm 250$ & & & 7920 B.C. \\
\hline-698 & $21: 154$ & $11,550 \pm 300$ & & & 9600 B.C. \\
\hline-699 & $21: 145$ & $1800 \pm 200$ & & & A.D. 150 \\
\hline-700 & $21: 145$ & $11,120 \pm 440$ & & & 9170 B.C. \\
\hline-701 & $21: 145$ & $<250$ & & & \\
\hline-702 & $21: 145$ & $2020 \pm 200$ & & & 70 B.C. \\
\hline .703 & $21: 159$ & $320 \pm 160$ & & & A.D. 1630 \\
\hline-704 & $21: 157$ & $32,500 \pm 1000$ & & & 30,550 B.C. \\
\hline-705 & $21: 176$ & $7580 \pm 250$ & & & 5630 B.C. \\
\hline .706 & $21: 164$ & $1950 \pm 200$ & & & A.D. 0 \\
\hline-707 & $21: 181$ & $1450 \pm 200$ & & & A.D. 500 \\
\hline-708 & $21: 179$ & $1.253^{3}$ & & & \\
\hline-710 & $21: 132$ & $12,700 \pm 300$ & & & 10,750 B.C. \\
\hline-712 & $21: 147$ & $>38,000$ & & & \\
\hline-714 & $21: 171$ & $9400 \pm 300$ & & & 7450 B.C. \\
\hline .715 & $21: 168$ & $4200 \pm 200$ & & & 2250 B.C. \\
\hline .716 & $21: 135$ & $5310 \pm 240$ & & & 3360 B.C. \\
\hline-717 & $21: 166$ & $6910 \pm 250$ & & & 4960 B.C. \\
\hline-719 & $21: 132$ & $>38,000$ & & & \\
\hline .720 & $21: 135$ & $9800 \pm 300$ & & & 7850 B.C. \\
\hline .721 & $21: 135$ & $<200$ & & & \\
\hline-722 & $21: 135$ & $<200$ & & & \\
\hline $\begin{array}{l}-723 \\
-724\end{array}$ & $\begin{array}{l}21: 152 \\
21: 152\end{array}$ & & & & $\begin{array}{l}9010 \text { B.C. } \\
17.150 \text { B C. }\end{array}$ \\
\hline $\begin{array}{l}-724 \\
-725\end{array}$ & $\begin{array}{l}21: 152 \\
21: 137\end{array}$ & $\begin{array}{r}19,100 \pm 300 \\
4030 \pm 150\end{array}$ & & & 2080 B.C. \\
\hline .726 & $21: 133$ & $6190 \pm 200$ & & & 4240 B.C. \\
\hline-727 & $21: 156$ & $1440 \pm 150$ & & & A.D. 510 \\
\hline-729 & $21: 137$ & $35,200 \pm 1000$ & & & 33,250 B.C. \\
\hline-730 & $21: 137$ & $17,100 \pm 360$ & & & 15,150 B.C. \\
\hline-731 & $21: 158$ & $29,700 \pm 1000$ & & & 27,750 B.C. \\
\hline-732 & $21: 173$ & $>35,000$ & & & \\
\hline-733 & $21: 174$ & $6820 \pm 200$ & & & 4870 B.C. \\
\hline-734 & $21: 156$ & $4830 \pm 200$ & & & 2880 B.C. \\
\hline-735 & $21: 133$ & $14,250 \pm 250$ & & & 12,300 B.C. \\
\hline-736 & $21: 173$ & $7740 \pm 200$ & & & 5790 B.C. \\
\hline-737 & $21: 130$ & $11,800 \pm 240$ & & & 9850 B.C. \\
\hline .738 & $21: 152$ & $18,750 \pm 300$ & & & 16,800 B.C. \\
\hline-739 & $21: 180$ & $3470 \pm 120$ & & & 1520 B.C. \\
\hline .741 & $21: 180$ & $2980 \pm 120$ & & & 1030 B.C. \\
\hline-743 & $21: 180$ & $3140 \pm 120$ & & & 1190 B.C. \\
\hline-744 & $21: 156$ & $10,690 \pm 300$ & & & 8740 B.C. \\
\hline
\end{tabular}




\begin{tabular}{|c|c|c|c|c|}
\hline $\begin{array}{l}\text { Laboratory } \\
\text { number }\end{array}$ & $\begin{array}{l}\text { Published } \\
\text { reference }\end{array}$ & $\begin{array}{l}\text { Original date or } \\
\text { other value }\end{array}$ & $\begin{array}{l}\text { Corrected date } \\
\text { or other value }\end{array}$ & $\partial \mathrm{C}^{14}, \%$ \\
\hline-745 & $21: 138$ & $23,500 \pm 400$ & & \\
\hline .746 & $21: 164$ & $>34,000$ & & \\
\hline-747 & $21: 154$ & $29,000 \pm 1000$ & & \\
\hline-748 & $21: 169$ & $13,500 \pm 400$ & & \\
\hline-749 & $21: 175$ & $480 \pm 200$ & & \\
\hline-750 & $21: 151$ & $9000 \pm 250$ & & \\
\hline-752 & $21: 151$ & $10,500 \pm 250$ & & \\
\hline-753 & $21: 173$ & $5650 \pm 200$ & & \\
\hline-754 & $21: 151$ & $420 \pm 160$ & & \\
\hline-755 & $21: 150$ & $8400 \pm 250$ & & \\
\hline .756 & $21: 173$ & $4250 \pm 250$ & & \\
\hline-757 & $21: 177$ & $>38,000$ & & \\
\hline .758 & $21: 155$ & $1320 \pm 200$ & & \\
\hline-759 & $21: 155$ & $510 \pm 160$ & & \\
\hline-760 & $21: 178$ & $990 \pm 180$ & & \\
\hline .762 & $21: 154$ & $12,220 \pm 250$ & & \\
\hline .763 & $21: 178$ & $3270 \pm 200$ & & \\
\hline-764 & $21: 178$ & $<200$ & & \\
\hline-765 & $21: 158$ & $7870 \pm 200$ & & \\
\hline .766 & $21: 170$ & $<200$ & & \\
\hline .767 & $21: 168$ & $10,250 \pm 250$ & & \\
\hline .768 & $21: 176$ & $2520 \pm 200$ & & \\
\hline .769 & $21: 176$ & $8100 \pm 250$ & & \\
\hline 770 & $21: 176$ & $4580 \pm 220$ & & \\
\hline-771 & $21: 149$ & $9800 \pm 250$ & & \\
\hline-773 & $21: 153$ & $>35,000$ & & \\
\hline-774 & $21: 158$ & $3060 \pm 200$ & & \\
\hline .775 & $21: 158$ & $7280 \pm 400$ & & \\
\hline .776 & $21: 164$ & $6600 \pm 400$ & & \\
\hline .777 & $21: 164$ & $6630 \pm 400$ & & \\
\hline-779 & $21: 164$ & $5950 \pm 400$ & & \\
\hline .780 & $21: 176$ & $2050 \pm 200$ & & \\
\hline .781 & $21: 179$ & $4740 \pm 160$ & & \\
\hline-782 & $21: 179$ & $7210 \pm 220$ & & \\
\hline-783 & $21: 179$ & $2950 \pm 200$ & & \\
\hline-785 & $21: 179$ & $2700 \pm 200$ & & \\
\hline .786 & $21: 160$ & $11,900 \pm 300$ & & \\
\hline .787 & $21: 159$ & $2200 \pm 250$ & & \\
\hline .788 & $21: 159$ & $28,000 \pm 1500$ & & \\
\hline .789 & $21: 159$ & $>32,000$ & & \\
\hline-790 & $21: 159$ & $17,800 \pm 450$ & & \\
\hline-791 & $21: 159$ & $>29,000$ & & \\
\hline .792 & $21: 160$ & $11,730 \pm 300$ & & \\
\hline .793 & $21: 156$ & $1040 \pm 200$ & & \\
\hline .794 & $21: 156$ & $6600 \pm 250$ & & \\
\hline-796 & $21: 160$ & $20,300 \pm 800$ & & \\
\hline .797 & $21: 160$ & $>30,000$ & & \\
\hline-798 & $21: 179$ & $1960 \pm 160$ & & \\
\hline-799 & $21: 145$ & $1100 \pm 170$ & & \\
\hline-800 & $21: 165$ & $6060 \pm 200$ & & \\
\hline-801 & $21: 153$ & $12,200 \pm 400$ & & \\
\hline-802 & $21: 157$ & $1800 \pm 160$ & & \\
\hline-803 & $21: 159$ & $>30,000^{4}$ & & \\
\hline-804 & $21: 159$ & $25,000 \pm 700^{5}$ & & \\
\hline-806 & $21: 173$ & $8720 \pm 300$ & & \\
\hline-807 & $21: 161$ & $18,500 \pm 600$ & & \\
\hline-808 & $21: 162$ & $29,000 \pm 1000$ & & \\
\hline-809 & $21: 173$ & $8000 \pm 300$ & & \\
\hline .810 & $21: 174$ & $>38,000$ & & \\
\hline-811 & $21: 164$ & $2030 \pm 240$ & & \\
\hline-812 & $21: 156$ & $<200$ & & \\
\hline
\end{tabular}

A.D.

B.C. date 21,550 B.C.

27,050 B.C. 11,550 B.C. A.D. 1470 7050 B.C. 8550 B.C. 3700 B.C. A.D. 1530 6450 B.C. 2300 B.C.

A.D. 630

A.D. 1440

A.D. 960

10,270 B.C. 1320 B.C.

5920 B.C.

8300 B.C.

570 B.C.

6150 B.C.

2630 B.C.

7850 B.C.

1110 B.C.

5330 B.C.

4650 B.C.

4680 B.C.

4000 B.C.

100 B.C.

2790 B.C.

5260 B.C.

1000 B.C.

750 B.C.

9950 B.C.

250 B.C.

26,050 B.C.

15,850 B.C.

9780 B.C.

A.D. 910

4650 B.C.

18,350 B.C.

10 B.C.

9150 B.C.

4110 B.C.

10,250 B.C.

A.D. 150

23,050 B.C. 6770 B.C.

16,550 B.C.

27,050 B.C. 6050 B.C.

80 B.C. 


\begin{tabular}{|c|c|c|c|c|}
\hline $\begin{array}{c}\text { Laboratory } \\
\text { number }\end{array}$ & $\begin{array}{l}\text { Published } \\
\text { reference }\end{array}$ & $\begin{array}{l}\text { Original date or } \\
\quad \text { other value }\end{array}$ & $\begin{array}{l}\text { Corrected date } \\
\text { or other value }\end{array}$ & $\partial \mathrm{C}^{14}, \%$ \\
\hline-813 & $21: 135$ & $8520 \pm 300$ & & \\
\hline-814 & $21: 142$ & $>38,000$ & & \\
\hline .815 & $21: 177$ & $3780 \pm 300$ & & \\
\hline .816 & $21: 177$ & $6100 \pm 300$ & & \\
\hline-817 & $21: 136$ & $>38,000$ & & \\
\hline-819 & $21: 160$ & $2620 \pm 200$ & & \\
\hline-820 & $21: 154$ & $10,420 \pm 300$ & & \\
\hline-822 & $21: 157$ & $700 \pm 200$ & & \\
\hline-823 & $21: 138$ & $4840 \pm 300$ & & \\
\hline-824 & $21: 149$ & $12,650 \pm 350$ & & \\
\hline-825 & $21: 149$ & $9300 \pm 350$ & & \\
\hline-826 & $21: 138$ & $>40,000$ & & \\
\hline-827 & $21: 149$ & $11,330 \pm 350$ & & \\
\hline-828 & $21: 129$ & $12.200 \pm 350$ & & \\
\hline-830 & $21: 166$ & $2230 \pm 250$ & & \\
\hline-831 & $21: 167$ & $>33,000$ & & \\
\hline-832 & $21: 142$ & $7030 \pm 260$ & & \\
\hline-833 & $21: 181$ & $2150 \pm 240$ & & \\
\hline-834 & $21: 181$ & $2200 \pm 240$ & & \\
\hline-835 & $21: 181$ & $760 \pm 500$ & & \\
\hline-836 & $21: 181$ & $2150 \pm 240$ & & \\
\hline-837 & $21: 181$ & $2080 \pm 240$ & & \\
\hline-838 & $21: 169$ & $1530 \pm 200$ & & \\
\hline-839 & $21: 170$ & $1060 \pm 200$ & & \\
\hline-841 & $21: 167$ & $5270 \pm 240$ & & \\
\hline-842 & $21: 168$ & $>38,000$ & & \\
\hline-843 & $21: 171$ & $31,300 \pm 1000$ & & \\
\hline-844 & $21: 167$ & $6330 \pm 240$ & & \\
\hline-846 & $21: 167$ & $<200$ & & \\
\hline-847 & $21: 170$ & $9100 \pm 260$ & & \\
\hline-848 & $21: 172$ & $11,390 \pm 300$ & & \\
\hline-849 & $21: 138$ & $23,700 \pm 550$ & & \\
\hline-851 & $21: 175$ & $660 \pm 300$ & & \\
\hline-853 & $21: 139$ & $25,500 \pm 600$ & & \\
\hline-854 & $21: 170$ & $<200$ & & \\
\hline-855 & $21: 158$ & $27,500 \pm 1000$ & & \\
\hline-856 & $21: 157$ & $3740 \pm 250$ & & \\
\hline-858 & $21: 161$ & $6640 \pm 250$ & & \\
\hline-859 & $21: 173$ & $5940 \pm 250$ & & \\
\hline-860 & $21: 175$ & $6200 \pm 320$ & & \\
\hline-861 & $21: 135$ & $12,660 \pm 400$ & & \\
\hline-862 & $21: 175$ & $6750 \pm 320$ & & \\
\hline-864 & $21: 162$ & $12,500 \pm 300$ & & \\
\hline-866 & $21: 136$ & $>38,000$ & & \\
\hline-867 & $21: 139$ & $22,200 \pm 450$ & & \\
\hline-868 & $21: 137$ & $27,500 \pm 900$ & & \\
\hline-869 & $21: 139$ & $37,000 \pm 1500$ & & \\
\hline-870 & $21: 138$ & $20,300 \pm 400$ & & \\
\hline-871 & $21: 139$ & $26,800 \pm 700$ & & \\
\hline-872 & $21: 150$ & $2520 \pm 200$ & & \\
\hline-873 & $21: 150$ & $10,550 \pm 300$ & & \\
\hline-875 & $21: 162$ & $40,000 \pm 2000$ & & \\
\hline-876 & $21: 162$ & $20,600 \pm 500$ & & \\
\hline-877 & $21: 136$ & $10,500 \pm 210$ & & \\
\hline-879 & $21: 145$ & $19,050 \pm 300$ & & \\
\hline-880 & $21: 145$ & $37,600 \pm 1500$ & & \\
\hline-881 & $21: 145$ & $14,300 \pm 250$ & & \\
\hline-882 & $22: 87$ & $11,600 \pm 200$ & & \\
\hline-883 & $22: 86$ & $12,080 \pm 300$ & & \\
\hline-885 & $22: 93$ & $>38,000$ & & \\
\hline-886 & $22: 93$ & $>38,000$ & & \\
\hline
\end{tabular}

A.D.) B.C. date 6570 B.C.

1830 B.C. 4150 B.C.

670 B.C.

8470 B.C.

A.D. 1250

2890 B.C.

10.700 B.C. 7350 B.C.

9380 B.C.

10,250 B.C.

280 B.C.

5080 B.C.

200 B.C.

250 B.C.

A.D. 1190

200 B.C.

130 B.C.

A.D. 420

A.D. 890

3320 B.C.

29,350 B.C. 4380 B.C.

7150 B.C.

9440 B.C.

21,750 B.C.

A.D. 1290

23,550 B.C.

25,550 B.C.

1790 B.C.

4690 B.C.

3990 B.C.

4250 B.C.

10,710 B.C.

4800 B.C.

10,550 B.C.

20,250 B.C.

25,550 B.C.

35,050 B.C.

18,350 B.C.

24,850 B.C.

570 B.C.

8600 B.C.

38,050 B.C.

18,650 B.C.

8550 B.C.

17,100 B.C.

35,650 B.C.

12,350 B.C.

9650 B.C.

10,130 B.C. 


\begin{tabular}{|c|c|c|c|c|}
\hline $\begin{array}{l}\text { Laboratory } \\
\text { number }\end{array}$ & $\begin{array}{l}\text { Published } \\
\text { reference }\end{array}$ & $\begin{array}{l}\text { Original date or } \\
\text { other value }\end{array}$ & $\begin{array}{l}\text { Corrected date } \\
\text { or other value }\end{array}$ & $\partial \mathrm{C}^{14}, \% 0$ \\
\hline-887 & $22: 96$ & $>33,000$ & & \\
\hline-888 & $22: 96$ & $>33,000$ & & \\
\hline-889 & $22: 96$ & $24,630 \pm 500$ & & \\
\hline-891 & $22: 93$ & $2520 \pm 200$ & & \\
\hline-892 & $22: 89$ & $12,390 \pm 400$ & & \\
\hline .894 & $22: 96$ & $22,500 \pm 500$ & & \\
\hline-896 & $22: 93$ & $4100 \pm 200$ & & \\
\hline-897 & $22: 96$ & $20,760 \pm 500$ & & \\
\hline-898 & $22: 90$ & $33,700 \pm 1000$ & & \\
\hline-899 & $22: 90$ & $13,900 \pm 400$ & & \\
\hline .900 & $22: 88$ & $10,080 \pm 280$ & & \\
\hline-901 & $22: 89$ & $30,800 \pm 1000$ & & \\
\hline-902 & $22: 90$ & $7460 \pm 250$ & & \\
\hline-903 & $22: 89$ & $29,000 \pm 900$ & & \\
\hline-904 & $22: 95$ & $20,400 \pm 500$ & & \\
\hline-905 & $22: 94$ & $17,360 \pm 650$ & & \\
\hline-907 & $22: 94$ & $10,140 \pm 300$ & & \\
\hline-908 & $22: 88$ & $<250$ & & \\
\hline .909 & $22: 94$ & $1910 \pm 250$ & & \\
\hline-910 & $22: 86$ & $>38,000$ & & \\
\hline-911 & $22: 94$ & $5290 \pm 250$ & & \\
\hline .912 & $22: 95$ & $9130 \pm 260$ & & \\
\hline-913 & $22: 94$ & $8170 \pm 300$ & & \\
\hline-914 & $22: 92$ & $11,330 \pm 320$ & & \\
\hline .915 & $22: 96$ & $10,720 \pm 300$ & & \\
\hline-916 & $22: 97$ & $4410 \pm 250$ & & \\
\hline .917 & $22: 87$ & $>40,000$ & & \\
\hline-918 & $22: 91$ & $600 \pm 250$ & & \\
\hline .919 & $22: 88$ & $10,850 \pm 300$ & & \\
\hline-921 & $22: 91$ & $>40,000$ & & \\
\hline-922 & $22: 91$ & $1640 \pm 250$ & & \\
\hline .925 & $22: 92$ & $350 \pm 250$ & & \\
\hline-926 & $22: 92$ & $350 \pm 250$ & & \\
\hline .929 & $124: 54$ & $>42,000$ & & \\
\hline .930 & $124: 59$ & $2550 \pm 200$ & & \\
\hline-932 & $124: 52$ & $>38,000$ & & \\
\hline-933 & $124: 50$ & $220 \pm 200$ & & \\
\hline-934 & $124: 50$ & $200 \pm 200$ & & \\
\hline-935 & $124: 50$ & $320 \pm 200$ & & \\
\hline-936 & $124: 50$ & $200 \pm 200$ & & \\
\hline .937 & $124: 67$ & $200 \pm 200$ & & \\
\hline-939 & $124: 49$ & $24,000 \pm 2000$ & & \\
\hline-940 & $124: 56$ & $11,640 \pm 275$ & & \\
\hline-941 & $124: 55$ & $20,300 \pm 500$ & & \\
\hline-942 & $124: 50$ & $11,800 \pm 1000$ & & \\
\hline-943 & $124: 55$ & $12,780 \pm 350$ & & \\
\hline .944 & $178: 375$ & $12,740 \pm 300$ & & \\
\hline .945 & $124: 37$ & $5900 \pm 200$ & & \\
\hline-947 & $124: 39$ & $11,950 \pm 350$ & & \\
\hline-948 & $124: 69$ & $15,400 \pm 400$ & & \\
\hline-949 & $124: 69$ & $>38,000$ & & \\
\hline-950 & $124: 59$ & $>38,000$ & & \\
\hline-951 & $178: 387$ & $8750 \pm 280$ & & \\
\hline .953 & $124: 73$ & $21,500 \pm 1500$ & & \\
\hline-954 & $124: 46$ & $9870 \pm 290$ & & \\
\hline .955 & $124: 73$ & $6300 \pm 1000$ & & \\
\hline-956 & $124: 46$ & $11,070 \pm 300$ & & \\
\hline-957 & $124: 47$ & $>38,000$ & & \\
\hline-958 & $124: 72$ & $6720 \pm 300$ & & \\
\hline-959 & $124: 72$ & $7680 \pm 300$ & & \\
\hline .960 & $124: 72$ & $7240 \pm 300$ & & \\
\hline
\end{tabular}

A.D./

B.C. date

22,680 B.C.

570 B.C.

10,440 B.C.

20,550 B.C.

2150 B.C.

18,810 B.C.

31,750 B.C.

11,950 B.C.

8130 B.C.

28,850 B.C.

5510 B.C.

27,050 B.C.

18,450 B.C.

15,410 B.C.

8190 B.C.

A.D. 40

3340 B.C.

7180 B.C.

6220 B.C.

9380 B.C.

8770 B.C.

2460 B.C.

A.D. 1350

8900 B.C.

A.D. 310

A.D. 1600

A.D. 1600

600 B.C.

A.D. 1730
A.D. 1750
A.D. 1630
A.D. 1750
A.D. 1750
22,050 B.C.
9690 B.C.
18,350 B.C.
9850 B.C.
10,830 B.C.
10,790 B.C.
3950 B.C.
10,000 B.C.
13,450 B.C.

6800 B.C.
19,550 B.C.
7920 B.C.
4350 B.C.
9120 B.C.

4770 B.C.

5730 B.C.

5290 B.C. 


\begin{tabular}{|c|c|c|c|c|}
\hline $\begin{array}{l}\text { Laboratory } \\
\text { number }\end{array}$ & $\begin{array}{l}\text { Published } \\
\text { reference }\end{array}$ & $\begin{array}{l}\text { Original date or } \\
\text { other value }\end{array}$ & $\begin{array}{l}\text { Corrected date } \\
\text { or other value }\end{array}$ & $\partial \mathrm{C}^{14}, \% 0$ \\
\hline-961 & $124: 38$ & $10,600 \pm 500$ & & \\
\hline-962 & $124: 44$ & $1780 \pm 250$ & & \\
\hline-963 & $124: 44$ & $4130 \pm 250$ & & \\
\hline-964 & $124: 43$ & $10,230 \pm 280$ & & \\
\hline-965 & $124: 43$ & $9600 \pm 280$ & & \\
\hline-967 & $124: 61$ & $4960 \pm 300$ & & \\
\hline-968 & $124: 66$ & $5160 \pm 300$ & & \\
\hline-969 & $124: 67$ & $>38,000$ & & \\
\hline .970 & $124: 40$ & $2420 \pm 250$ & & \\
\hline-971 & $124: 40$ & $2800 \pm 250$ & & \\
\hline-973 & $124: 40$ & $3660 \pm 250$ & & \\
\hline-974 & $124: 46$ & $11,650 \pm 310$ & & \\
\hline-975 & $124: 67$ & $9720 \pm 320$ & & \\
\hline-976 & $124: 66$ & $>42,000$ & & \\
\hline-977 & $124: 62$ & $>40,000$ & & \\
\hline $.978^{6}$ & $124: 61$ & $2000 \pm 250$ & & \\
\hline$-978^{6}$ & $124: 69$ & $2000 \pm 250$ & & \\
\hline-979 & $124: 66$ & $5380 \pm 260$ & & \\
\hline$-980^{6}$ & $124: 64$ & $10,230 \pm 300$ & & \\
\hline$-980^{6}$ & $124: 68$ & $10,230 \pm 300$ & & \\
\hline-981 & $124: 51$ & $5070 \pm 250$ & & \\
\hline-982 & $124: 54$ & $18,900 \pm 500$ & & \\
\hline-983 & $124: 48$ & $10,350 \pm 350$ & & \\
\hline-984 & $124: 57$ & $12,090 \pm 350$ & & \\
\hline-985 & $124: 65$ & $6960 \pm 290$ & & \\
\hline-987 & $124: 48$ & $12,530 \pm 350$ & & \\
\hline-988 & $124: 40$ & $1700 \pm 300$ & & \\
\hline .989 & $124: 52$ & $<200$ & & \\
\hline-990 & $124: 46$ & $>38,000$ & & \\
\hline-991 & $124: 41$ & $12,170 \pm 300$ & & \\
\hline .992 & $124: 39$ & $>38,000$ & & \\
\hline-993 & $124: 45$ & $9900 \pm 400$ & & \\
\hline-995 & $124: 59$ & $10,230 \pm 600$ & & \\
\hline-996 & $124: 56$ & $11,660 \pm 350$ & & \\
\hline-997 & $124: 58$ & $>38.000$ & & \\
\hline-998 & $124: 59$ & $20,800 \pm 1200$ & & \\
\hline-999 & $124: 45$ & $>38,000$ & & \\
\hline-1000 & $124: 59$ & $8600 \pm 300$ & & \\
\hline-1002 & $124: 70$ & $>38,000$ & & \\
\hline-1005 & $124: 47$ & $10,050 \pm 300$ & & \\
\hline-1008 & $124: 70$ & $>38,000$ & & \\
\hline-1009 & $124: 72$ & $>38,000$ & & \\
\hline-1010 & $124: 72$ & $>35,000$ & & \\
\hline-1011 & $124: 38$ & $12,800 \pm 450$ & & \\
\hline-1016 & $124: 74$ & $4800 \pm 300$ & & \\
\hline-1017 & $124: 48$ & $6340 \pm 300$ & & \\
\hline -1018 & $124: 52$ & $900 \pm 250$ & & \\
\hline -1019 & $124: 46$ & $9000 \pm 300$ & & \\
\hline-1020 & $124: 47$ & $>38,000$ & & \\
\hline-1021 & $124: 45$ & $>38,000$ & & \\
\hline-1023 & $124: 70$ & $>35,000$ & & \\
\hline-1028 & $124: 44$ & $>38,000$ & & \\
\hline-1029 & $124: 57$ & $>38,000$ & & \\
\hline-1030 & $124: 57$ & $4700 \pm 300$ & & \\
\hline-1031 & $124: 47$ & $5950 \pm 300$ & & \\
\hline-1033 & $124: 48$ & $10,060 \pm 300$ & & \\
\hline-1034 & $124: 50$ & $>35,000$ & & \\
\hline-1035 & $124: 73$ & $8500 \pm 600$ & & \\
\hline-1036 & $124: 73$ & $3300 \pm 300$ & & \\
\hline-1037 & $124: 55$ & $11,600 \pm 400$ & & \\
\hline-1038 & $124: 41$ & $10,450 \pm 400$ & & \\
\hline
\end{tabular}

A.D./

B.C. date 8650 B.C.

A.D. 170

2180 B.C.

8280 B.C.

7650 B.C.

3010 B.C.

3210 B.C.

470 B.C.

850 B.C.

1710 B.C.

9700 B.C.

7770 B.C.

50 B.C.

50 B.C.

3430 B.C.

8280 B.C.

8280 B.C.

3120 B.C.

16,950 B.C.

8400 B.C.

10,140 B.C.

5010 B.C.

10,580 B.C.

A.D. 250

10,220 B.C.

7950 B.C.

8280 B.C.

9710 B.C.

18,850 B.C.

6650 B.C.

8100 B.C.

10,850 B.C.

2850 B.C.

4390 B.C.

A.D. 1050

7050 B.C.

2750 B.C.

4000 B.C.

8110 B.C.

6550 B.C.

1350 B.C.

9650 B.C.

8500 B.C. 


\begin{tabular}{|c|c|c|c|c|c|}
\hline $\begin{array}{l}\text { Laboratory } \\
\text { number }\end{array}$ & $\begin{array}{l}\text { Published } \\
\text { reference }\end{array}$ & $\begin{array}{l}\text { Original date or } \\
\text { other value }\end{array}$ & $\begin{array}{l}\text { Corrected date } \\
\text { or other value }\end{array}$ & $\partial \mathrm{C}^{14}, \%$ & $\begin{array}{l}\text { A.D./ } \\
\text { B.C. date }\end{array}$ \\
\hline-1039 & $124: 47$ & $2600 \pm 300$ & & & 650 B.C. \\
\hline-1040 & $124: 43$ & $9140 \pm 400$ & & & 7190 B.C. \\
\hline-1041 & $124: 42$ & $1550 \pm 300$ & & & A.D. 400 \\
\hline -1042 & $124: 42$ & $2100 \pm 300$ & & & 150 B.C. \\
\hline-1044 & $124: 42$ & $<200$ & & & \\
\hline-1045 & $124: 45$ & $28,700 \pm 800$ & & & 26,750 B.C. \\
\hline-1046 & $124: 72$ & $4030 \pm 350$ & & & 2080 B.C. \\
\hline-1047 & $124: 72$ & $600 \pm 250$ & & & A.D. 1350 \\
\hline -1048 & $124: 43$ & $<200$ & & & \\
\hline-1050 & $178: 384$ & $13,350 \pm 400$ & & & 11,400 B.C. \\
\hline-1051 & $178: 385$ & $13,200 \pm 400$ & & & 11,250 B.C. \\
\hline-1052 & $178: 385$ & $12,700 \pm 400$ & & & 10,750 B.C. \\
\hline-1053 & $124: 43$ & $<200$ & & & \\
\hline-1054 & $124: 42$ & $>37,000$ & & & \\
\hline-1055 & $124: 43$ & $17,950 \pm 550$ & & & 16,000 B.C. \\
\hline-1057 & $124: 44$ & $9200 \pm 600$ & & & 7250 B.C. \\
\hline-1058 & $124: 45$ & $11,250 \pm 400$ & & & 9300 B.C. \\
\hline-1059 & $124: 44$ & $11,500 \pm 600$ & & & 9550 B.C. \\
\hline-1060 & $124: 60$ & $9500 \pm 400$ & & & 7550 B.C. \\
\hline-1061 & $178: 388$ & $1520 \pm 300$ & & & A.D. 430 \\
\hline-1063 & $124: 70$ & $6035 \pm 300$ & & & 4085 B.C. \\
\hline-1064 & $124: 39$ & $<250$ & & & \\
\hline-1065 & $124: 39$ & $<250$ & & & \\
\hline-1066 & $124: 71$ & $4200 \pm 320$ & & & 2250 B.C. \\
\hline-1067 & $124: 71$ & $6100 \pm 1000$ & & & 4.150 B.C. \\
\hline-1069 & $124: 49$ & $1380 \pm 250$ & & & A.D. 570 \\
\hline-1070 & $124: 60$ & $11,940 \pm 500$ & & & 9990 B.C. \\
\hline-1071 & $178: 389$ & $15,300 \pm 500$ & & & 13,350 B.C. \\
\hline-1072 & $124: 70$ & $6650 \pm 600$ & & & 4700 B.C. \\
\hline-1073 & $124: 71$ & $4970 \pm 260$ & & & 3020 B.C. \\
\hline-1075 & $124: 66$ & $440 \pm 250$ & & & A.D. 1510 \\
\hline-1076 & $124: 71$ & $6880 \pm 300$ & & & 4930 B.C. \\
\hline-1078 & $178: 389$ & $13,980 \pm 700$ & & & 12,030 B.C. \\
\hline-1082 & $124: 38$ & $2850 \pm 260$ & & & 900 B.C. \\
\hline-1083 & $124: 71$ & $7060 \pm 300$ & & & 5110 B.C. \\
\hline-1084 & $124: 71$ & $5980 \pm 300$ & & & 4030 B.C. \\
\hline-1086 & $124: 68$ & $3510 \pm 250$ & & & 1560 B.C. \\
\hline-1088 & $124: 71$ & $1200 \pm 300$ & & & A.D. 750 \\
\hline-1089 & $124: 61$ & $4170 \pm 250$ & & & 2220 B.C. \\
\hline-1090 & $124: 71$ & $7730 \pm 400$ & & & 5780 B.C. \\
\hline -1091 & $124: 58$ & $20,350 \pm 600$ & & & 18,400 B.C. \\
\hline-1092 & $124: 40$ & $3400 \pm 300$ & & & 1450 B.C. \\
\hline -1093 & $124: 40$ & $4860 \pm 350$ & & & 2910 B.C. \\
\hline -1094 & $124: 40$ & $1040 \pm 300$ & & & A.D. 910 \\
\hline-1095 & $124: 40$ & $1850 \pm 300$ & & & A.D. 100 \\
\hline-1096 & $124: 40$ & $2240 \pm 300$ & & & 290 B.C. \\
\hline -1098 & $124: 40$ & $3060 \pm 300$ & & & 1110 B.C. \\
\hline -1099 & $124: 40$ & $3170 \pm 300$ & & & 1220 B.C. \\
\hline-1100 & $124: 74$ & $-312 \pm 75 \%$ & & $-312 \pm 75$ & $3000 \pm 600$ \\
\hline-1101 & $124: 74$ & $-75 \%$ & & -75 & $<600$ \\
\hline-1103 & $124: 56$ & $>38,000$ & & & \\
\hline-1106 & $124: 67$ & $<200$ & & & \\
\hline-1108 & $124: 67$ & $6730 \pm 260$ & & & 4780 B.C. \\
\hline-1109 & $124: 41$ & $10,560 \pm 350$ & & & 8610 B.C. \\
\hline-1110 & $124: 48$ & $11,640 \pm 350$ & & & 9690 B.C. \\
\hline-1111 & $124: 67$ & $<200$ & & & \\
\hline-1112 & $124: 55$ & $5020 \pm 260$ & & & 3070 B.C. \\
\hline-1113 & $124: 67$ & $>38,000$ & & & \\
\hline-1114 & $124: 57$ & $2660 \pm 250$ & & & 710 B.C. \\
\hline-1115 & $124: 59$ & $3500 \pm 250$ & & & 1550 B.C. \\
\hline-1116 & $124: 57$ & $4000 \pm 250$ & & & 2050 B.C. \\
\hline
\end{tabular}




\begin{tabular}{|c|c|c|c|c|}
\hline $\begin{array}{c}\text { Laboratory } \\
\text { number }\end{array}$ & $\begin{array}{l}\text { Published } \\
\text { reference }\end{array}$ & $\begin{array}{l}\text { Original date or } \\
\text { other value }\end{array}$ & $\begin{array}{l}\text { Corrected date } \\
\text { or other value }\end{array}$ & $\partial \mathrm{C}^{14}, \%$ \\
\hline-1118 & $124: 56$ & $2980 \pm 250$ & & \\
\hline-1119 & $124: 57$ & $320 \pm 200$ & & \\
\hline-1120 & $124: 56$ & $290 \pm 200$ & & \\
\hline-1121 & $124: 54$ & $33,000 \pm 1600$ & & \\
\hline-1125 & $124: 53$ & $27,000 \pm 900$ & & \\
\hline-1128 & $124: 53$ & $34,000 \pm 1600$ & & \\
\hline-1129 & $124: 51$ & $4150 \pm 300$ & & \\
\hline-1132 & $124: 37$ & $>38,000$ & & \\
\hline-1133 & $124: 52$ & $1500 \pm 250$ & & \\
\hline-1134 & $124: 65$ & $17,600 \pm 400$ & & \\
\hline-1135 & $124: 55$ & $4900 \pm 300$ & & \\
\hline$-1136^{7}$ & $124: 52$ & $7830 \pm 350$ & & \\
\hline-1138 & $124: 49$ & $6070 \pm 320$ & & \\
\hline-1139 & $124: 49$ & $6040 \pm 350$ & & \\
\hline-1141 & $124: 44$ & $11,560 \pm 4.00$ & & \\
\hline-1144 & $124: 42$ & $>38,000$ & & \\
\hline-1150 & $124: 44$ & $9500 \pm 350$ & & \\
\hline-1151 & $124: 44$ & $16,700 \pm 420$ & & \\
\hline-1153 & $178: 373$ & $9870 \pm 370$ & & \\
\hline-1154 & $124: 62$ & $4470 \pm 300$ & & \\
\hline-1155 & $124: 61$ & $3500 \pm 300$ & & \\
\hline-1156 & $124: 69$ & $1420 \pm 280$ & & \\
\hline-1157 & $124: 62$ & $<200$ & & \\
\hline-1159 & $124: 63$ & $4300 \pm 300$ & & \\
\hline-1160 & $124: 54$ & $33,000 \pm 1500$ & & \\
\hline-1161 & $124: 63$ & $9650 \pm 370$ & & \\
\hline-1162 & $124: 65$ & $>42,000$ & & \\
\hline-1163 & $124: 61$ & $7450 \pm 400$ & & \\
\hline-1164 & $124: 65$ & $7880 \pm 400$ & & \\
\hline-1165 & $124: 64$ & $1630 \pm 350$ & & \\
\hline-1166 & $124: 72$ & $1650 \pm 350$ & & \\
\hline-1167 & $124: 66$ & $6200 \pm 300$ & & \\
\hline-1168 & $124: 65$ & $>38,000$ & & \\
\hline-1169 & $124: 65$ & $2000 \pm 300$ & & \\
\hline-1170 & $124: 67$ & $6930 \pm 300$ & & \\
\hline-1171 & $124: 68$ & $1550 \pm 300$ & & \\
\hline-1173 & $124: 68$ & $6170 \pm 300$ & & \\
\hline-1174 & $124: 66$ & $25,800 \pm 800$ & & \\
\hline-1175 & $124: 61$ & $1650 \pm 300$ & & \\
\hline-1177 & $124: 54$ & $35,000 \pm 3000$ & & \\
\hline-1180 & $124: 60$ & $7660 \pm 300$ & & \\
\hline-1181 & $124: 58$ & $22,400 \pm 800$ & & \\
\hline-1182 & $124: 58$ & $24,100 \pm 900$ & & \\
\hline-1183 & $124: 49$ & $12,060 \pm 700$ & & \\
\hline-1184 & $178: 378$ & $23,400 \pm 800$ & & \\
\hline-1185 & $178: 378$ & $2540 \pm 300$ & & \\
\hline-1186 & $124: 58$ & $18,100 \pm 700$ & & \\
\hline-1187 & $178: 373$ & $15,300 \pm 800$ & & \\
\hline-1189 & $124: 48$ & $12,050 \pm 400$ & & \\
\hline-1191 & $124: 53$ & $2050 \pm 300$ & & \\
\hline-1192 & $124: 51$ & $>38,000$ & & \\
\hline-1199 & $124: 60$ & $2080 \pm 300$ & & \\
\hline-1200 & $124: 51$ & $>38,000$ & & \\
\hline-1201 & $124: 50$ & $13,300 \pm 500$ & & \\
\hline-1202 & $124: 63$ & $700 \pm 250$ & & \\
\hline-1205 & $124: 63$ & $600 \pm 250$ & & \\
\hline .1206 & $124: 64$ & $8200 \pm 300$ & & \\
\hline-1207 & $124: 65$ & $10,150 \pm 400$ & & \\
\hline-1209 & $124: 68$ & $3120 \pm 250$ & & \\
\hline-1210 & $124: 63$ & $2560 \pm 250$ & & \\
\hline-1211 & $178: 396$ & $5580 \pm 300$ & & \\
\hline
\end{tabular}

A.D./

B.C. date 1030 B.C. A.D. 1630 A.D. 1660 31,050 B.C. 25,050 B.C. 32,050 B.C. 2200 B.C.

A.D. 450

15,650 B.C. 2950 B.C.

5880 B.C.

4120 B.C.

4090 B.C.

9610 B.C.

7550 B.C.

14,750 B.C. 7920 B.C.

2520 B.C.

1550 B.C.

A.D. 530

2350 B.C.

31,050 B.C.

7700 B.C.

5500 B.C.

5930 B.C.

A.D. 320

A.D. 300

4250 B.C.

50 B.C.

4980 B.C.

A.D. 400

4220 B.C.

23,850 B.C.

A.D. 300

33,050 B.C.

5710 B.C.

20,450 B.C.

22,150 B.C.

10,110 B.C.

21,450 B.C.

590 B.C.

16,150 B.C.

13,350 B.C.

10,100 B.C.

100 B.C.

130 B.C.

11,350 B.C.

A.D. 1250

A.D. 1350

6250 B.C.

8200 B.C.

1170 B.C.

610 B.C.

3630 B.C. 


\begin{tabular}{|c|c|c|c|c|}
\hline $\begin{array}{l}\text { Laboratory } \\
\text { number }\end{array}$ & $\begin{array}{l}\text { Published } \\
\text { reference }\end{array}$ & $\begin{array}{l}\text { Original date or } \\
\text { other value }\end{array}$ & $\begin{array}{l}\text { Corrected date } \\
\text { or other value }\end{array}$ & $\partial \mathrm{C}^{14}, \% 0$ \\
\hline-1212 & $124: 68$ & $12,400 \pm 450$ & & \\
\hline-1213 & $124: 62$ & $9990 \pm 400$ & & \\
\hline .1221 & $124: 53$ & $32,500 \pm 1500$ & & \\
\hline-1222 & $124: 38$ & $3670 \pm 300$ & & \\
\hline-1223 & $124: 38$ & $2780 \pm 250$ & & \\
\hline-1225 & $124: 53$ & $>39,000$ & & \\
\hline-1226 & $124: 53$ & $<200$ & & \\
\hline-1227 & $178: 386$ & $15,000 \pm 400$ & & \\
\hline-1229 & $124: 38$ & $400 \pm 200$ & & \\
\hline-1230 & $178: 374$ & $10,650 \pm 300$ & & \\
\hline-1231 & $178: 374$ & $6300 \pm 300$ & & \\
\hline-1232 & $178: 377$ & $>40,000$ & & \\
\hline-1233 & $178: 376$ & $22,000 \pm 600$ & & \\
\hline-1235 & $178: 390$ & $7400 \pm 300$ & & \\
\hline-1240 & $178: 397$ & $1850 \pm 350$ & & \\
\hline-1241 & $178: 372$ & $8600 \pm 400$ & & \\
\hline-1242 & $178: 372$ & $9500 \pm 400$ & & \\
\hline-1243 & $178: 372$ & $8520 \pm 400$ & & \\
\hline-1244 & $178: 389$ & $7670 \pm 350$ & & \\
\hline-1245 & $178: 372$ & $9370 \pm 400$ & & \\
\hline .1246 & $124: 69$ & $3890 \pm 300$ & & \\
\hline-1247 & $124: 69$ & $7010 \pm 350$ & & \\
\hline-1249 & $178: 394$ & $8550 \pm 400$ & & \\
\hline-1250 & $178: 394$ & $7270 \pm 350$ & & \\
\hline-1251 & $178: 395$ & $>42,000$ & & \\
\hline-1252 & $178: 395$ & $>42,000$ & & \\
\hline-1253 & $178: 395$ & $>42,000$ & & \\
\hline-1254 & $178: 394$ & $11,340 \pm 400$ & & \\
\hline-1255 & $178: 394$ & $9020 \pm 350$ & & \\
\hline-1256 & $178: 394$ & $>38,000$ & & \\
\hline .1257 & $178: 394$ & $>38,000$ & & \\
\hline-1259 & $178: 393$ & $660 \pm 250$ & & \\
\hline-1261 & $178: 393$ & $460 \pm 250$ & & \\
\hline-1262 & $178: 394$ & $34,000 \pm 2000$ & & \\
\hline-1269 & $178: 375$ & $850 \pm 200$ & & \\
\hline-1270 & $178: 375$ & $>42,000$ & & \\
\hline-1271 & $178: 375$ & $12,820 \pm 400$ & & \\
\hline-1272 & $178: 395$ & $2420 \pm 200$ & & \\
\hline-1273 & $178: 389$ & $>33,000$ & & \\
\hline-1281 & $178: 375$ & $>42,000$ & & \\
\hline-1284 & $178: 388$ & $800 \pm 200$ & & \\
\hline-1286 & $178: 388$ & $1560 \pm 200$ & & \\
\hline$-1287^{8}$ & $124: 60$ & $>34,000$ & & \\
\hline-1290 & $124: 59$ & $>40,000$ & & \\
\hline .1291 & $178: 385$ & $<200$ & & \\
\hline-1292 & $178: 376$ & $>40,000$ & & \\
\hline .1293 & $178: 376$ & $>36,000$ & & \\
\hline-1294 & $178: 376$ & $>40,000$ & & \\
\hline-1299 & $178: 389$ & $4200 \pm 300$ & & \\
\hline .1300 & $178: 376$ & $>40,000$ & & \\
\hline-1301 & $178: 390$ & $6000 \pm 600$ & & \\
\hline-1302 & $178: 390$ & $7880 \pm 500$ & & \\
\hline-1303 & $178: 390$ & $18,700 \pm 600$ & & \\
\hline-1304 & $178: 395$ & $5850 \pm 320$ & & \\
\hline-1305 & $178: 386$ & $15,100 \pm 600$ & & \\
\hline-1306 & $178: 373$ & $7390 \pm 500$ & & \\
\hline-1313 & 178:391 & $<200$ & & \\
\hline-1314 & $178: 391$ & $<200$ & & \\
\hline-1315 & $178: 391$ & $<200$ & & \\
\hline-1317 & 178:381 & $12,000 \pm 400$ & & \\
\hline-1318 & $178: 381$ & $12,200 \pm 450$ & & \\
\hline
\end{tabular}

A.D./

B.C. date

10,450 B.C.

8040 B.C.

30,550 B.C.

1720 B.C.

830 B.C.

13,050 B.C.

A.D. 1550

8700 B.C.

4350 B.C.

20,050 B.C.

5450 B.C.

A.D. 100

6650 B.C.

7550 B.C.

6570 B.C.

5720 B.C.

7420 B.C.

1940 B.C.

5060 B.C.

6600 B.C.

5320 B.C.

9390 B.C.

7070 B.C.

A.D. 1290

A.D. 1490

32,050 B.C.

A.D. 1100

10,870 B.C.

470 B.C.

A.D. 1150

A.D. 390

2250 B.C.

4050 B.C.

7930 B.C.

16,750 B.C.

3900 B.C.

13,150 B.C.

5440 B.C.

10,050 B.C.

10,250 B.C. 


\begin{tabular}{|c|c|c|c|c|}
\hline $\begin{array}{l}\text { Laboratory } \\
\text { number }\end{array}$ & $\begin{array}{l}\text { Published } \\
\text { reference }\end{array}$ & $\begin{array}{l}\text { Original date or } \\
\quad \text { other value }\end{array}$ & $\begin{array}{l}\text { Corrected date } \\
\text { or other value }\end{array}$ & $\begin{array}{l}\text { A.D./ } \\
\text { B.C. date }\end{array}$ \\
\hline-1319 & $178: 393$ & $610 \pm 250$ & & A.D. 1340 \\
\hline-1321 & $178: 380$ & $32,500 \pm 2000$ & & 30,550 B.C. \\
\hline-1322 & $178: 380$ & $10,230 \pm 300$ & & 8280 B.C. \\
\hline-1323 & 178:381 & $13,700 \pm 350$ & & 11,750 B.C. \\
\hline-1324 & $178: 380$ & $22,500 \pm 600$ & & 20,550 B.C. \\
\hline-1325 & $178: 381$ & $12,110 \pm 300$ & & 10,160 B.C. \\
\hline-1326 & $178: 391$ & $5250 \pm 300$ & & 3300 B.C. \\
\hline-1327 & $178: 380$ & $11,730 \pm 350$ & & 9780 B.C. \\
\hline-1328 & $178: 385$ & $2800 \pm 250$ & & 850 B.C. \\
\hline-1336 & $178: 395$ & $7380 \pm 250$ & & 5430 B.C. \\
\hline-1337 & 178:391 & $>38,000$ & & \\
\hline-1338 & $178: 384$ & $12,090 \pm 300$ & & 10,140 B.C. \\
\hline-1339 & $178: 384$ & $10,190 \pm 250$ & & 8240 B.C. \\
\hline-1340 & $178: 384$ & $1850 \pm 200$ & & A.D. 100 \\
\hline-1341 & $178: 396$ & $4880 \pm 250$ & & 2930 B.C. \\
\hline-1342 & $178: 373$ & $0 \pm 200$ & & A.D. 1950 \\
\hline-1343 & $178: 391$ & $28,300 \pm 1000$ & & 26,350 B.C. \\
\hline-1353 & $178: 375$ & $21,080 \pm 400$ & & 19,130 B.C. \\
\hline-1357 & $178: 374$ & $<200$ & & \\
\hline-1358 & $178: 374$ & $10,600 \pm 250$ & & 8650 B.C. \\
\hline-1359 & $178: 385$ & $4170 \pm 250$ & & 2220 B.C. \\
\hline-1360 & $178: 378$ & $9810 \pm 300$ & & 7860 B.C. \\
\hline-1361 & $178: 378$ & $9820 \pm 300$ & & 7870 B.C. \\
\hline-1364 & 178:389 & $9440 \pm 300$ & & 7490 B.C. \\
\hline-1366 & $178: 384$ & $1945 \pm 250$ & & A.D. 5 \\
\hline-1367 & $178: 390$ & $3750 \pm 300$ & & 1800 B.C. \\
\hline-1369 & $178: 377$ & $9860 \pm 400$ & & 7910 B.C. \\
\hline-1370 & $178: 379$ & $>33,000$ & & \\
\hline-1372 & $178: 378$ & $12,200 \pm 400$ & & 10,250 B.C. \\
\hline 1373 & $178: 379$ & $14,000 \pm 500$ & & 12,050 B.C. \\
\hline-1374 & $178: 379$ & $>32,000$ & & \\
\hline-1376 & $178: 383$ & $10,200 \pm 300$ & & 8250 B.C. \\
\hline-1377 & $178: 392$ & $11,190 \pm 300$ & & 9240 B.C. \\
\hline-1378 & $178: 396$ & $1490 \pm 250$ & & A.D. 460 \\
\hline-1379 & $178: 392$ & $>38,000$ & & \\
\hline-1381 & $178: 396$ & $7900 \pm 350$ & & 5950 B.C. \\
\hline-1386 & $178: 388$ & $11,100 \pm 300$ & & 9150 B.C. \\
\hline-1388 & $178: 388$ & $24,300 \pm 700$ & & 22,350 B.C. \\
\hline-1389 & $178: 374$ & $>38,000$ & & \\
\hline-1390 & $178: 392$ & $>38,000$ & & \\
\hline-1391 & $178: 379$ & $11,130 \pm 600$ & & 9180 B.C. \\
\hline-1392 & $178: 379$ & $10,990 \pm 600$ & & 9040 B.C. \\
\hline-1393 & $178: 388$ & $2040 \pm 200$ & & 90 B.C. \\
\hline-1394 & $178: 388$ & $2460 \pm 200$ & & 510 B.C. \\
\hline 1396 & $178: 387$ & $2340 \pm 200$ & & 390 B.C. \\
\hline-1397 & 178:386 & $1500 \pm 200$ & & A.D. 450 \\
\hline-1398 & $178: 385$ & $250 \pm 200$ & & A.D. 1700 \\
\hline-1404 & $178: 392$ & $2390 \pm 200$ & & 440 B.C. \\
\hline-1405 & $178: 392$ & $4470 \pm 250$ & & 2520 B.C. \\
\hline-1406 & $178: 382$ & $8140 \pm 300$ & & 6190 B.C. \\
\hline-1407 & $178: 383$ & $8030 \pm 300$ & & 6080 B.C. \\
\hline-1408 & 178:383 & $2800 \pm 300$ & & 850 B.C. \\
\hline-1413 & $178: 383$ & $>32,000$ & & \\
\hline-1414 & $178: 376$ & $10,230 \pm 500$ & & 8280 B.C. \\
\hline-1415 & 178:395 & $1460 \pm 200$ & & A.D. 490 \\
\hline-1416 & 178:394 & $4740 \pm 250$ & & 2790 B.C. \\
\hline-1417 & $178: 383$ & $1440 \pm 250$ & & A.D. 510 \\
\hline-1418 & $178: 381$ & $11,720 \pm 500$ & & 9770 B.C. \\
\hline-1419 & $178: 382$ & $1700 \pm 250$ & & A.D. 250 \\
\hline-1420 & $178: 382$ & $25,000 \pm 800$ & & 23,050 B.C. \\
\hline 1421 & $178: 382$ & $1820 \pm 250$ & & A.D. 130 \\
\hline
\end{tabular}




\begin{tabular}{cccccc}
$\begin{array}{c}\text { Laboratory } \\
\text { number }\end{array}$ & $\begin{array}{c}\text { Published } \\
\text { reference }\end{array}$ & $\begin{array}{c}\text { Original date or } \\
\text { other value }\end{array}$ & $\begin{array}{c}\text { Corrected date } \\
\text { or other value }\end{array}$ & $\partial \mathrm{C}^{14}, \% 0$ & $\begin{array}{c}\text { A.D./ } \\
\text { B.C. date }\end{array}$ \\
-1422 & $178: 381$ & $27,400 \pm 800$ & & \\
-1424 & $178: 393$ & $>38,000$ & & \\
-1426 & $178: 396$ & $>38,000$ & & \\
-1427 & $178: 393$ & $31,000 \pm 800$ & & 29,050 B.C. \\
-1428 & $178: 379$ & $>42,000$ & \\
-1429 & $178: 386$ & $>38,000$ & \\
-1432 & $178: 378$ & $210 \pm 200$ & A.D. 1740 \\
-1433 & $178: 377$ & $>38,000$ & \\
-1434 & $178: 377$ & $10,100 \pm 300$ & 8150 B.C. \\
-1436 & $178: 377$ & $9990 \pm 300$ & 8040 B.C. \\
-1437 & $178: 387$ & $34,000 \pm 800$ & 32,050 B.C. \\
-1458 & $178: 387$ & $>34,000$ & \\
-1459 & $178: 387$ & $>38,000$ & & \\
-1460 & $178: 396$ & $8450 \pm 350$ & & \\
\hline
\end{tabular}

${ }^{1}$ Reference standard for early samples was 19th-century wood, not significantly different (ref. 22, p. 86) from $0.95 \times$ NBS oxalic acid.

${ }^{2}$ Listed originally as "possibly older".

${ }^{3}$ Ratio, activity of sample to activity of wood standard.

${ }^{4}$ Less than $2 \%$ of modern activity.

${ }^{5} 4.2 \%$ of modern activity.

${ }^{\circ}$ Sample published twice with different titles.

${ }^{7}$ Published, by typographic error, as W-1135.

${ }^{8}$ Also published in ref. 178 , p. 390 .

\section{University of Wisconsin, Center for Climatic Research}

$\begin{array}{ccccc}\begin{array}{c}\text { Laboratory } \\ \text { number }\end{array} & \begin{array}{c}\text { Published } \\ \text { reference }\end{array} & \begin{array}{c}\text { Original date or } \\ \text { other value }\end{array} & \begin{array}{c}\text { Corrected date } \\ \text { or other value }\end{array} & \partial \mathrm{C}^{14}, \% \text { A.D./ } \\ \text { WIS-1 } & 179: 405 & 2380 \pm 90 & \text { B.C. date } \\ -2^{1} & 179: 400 & 12,800 \pm 450 & 430 \text { B.C. } \\ -3 & 179: 400 & 820 \pm 80 & \text { 10,850 B.C. } \\ -4^{2} & 179: 400 & 5290 \pm 120 & \text { A.D. } 1130 \\ -5 & 179: 405 & 880 \pm 180 & \text { 3340 B.C. } \\ -6 & 179: 406 & 870 \pm 100 & \text { A.D. } 1070 \\ -7 & 179: 406 & 4000 \pm 160 & \text { A.D. } 1080 \\ -8 & 179: 400 & 1000 \pm 70 & 2050 \text { B.C. } \\ -9 & 179: 401 & 1020 \pm 80 & \text { A.D. } 950 \\ -10 & 179: 401 & 900 \pm 90 & \text { A.D. } 930 \\ -11 & 179: 401 & 960 \pm 100 & \text { A.D. } 1050 \\ -12 & 179: 406 & 3430 \pm 110 & \text { A.D. } 990 \\ -13 & 179: 401 & 1140 \pm 100 & 1480 \text { B.C. } \\ -14 & 179: 401 & 850 \pm 90 & \text { A.D. } 810 \\ -15 & 179: 406 & 1450 \pm 90 & \text { A.D. } 1100 \\ -16 & 179: 401 & 690 \pm 90 & \text { A.D. } 500 \\ -17 \mathrm{a} & 179: 406 & 1140 \pm 90 & \text { A.D. } 1260 \\ -17 \mathrm{~b} & 179: 406 & 1050 \pm 180 & \text { A.D. } 810 \\ -18 & 179: 406 & 3550 \pm 120 & \text { A.D. } 900 \\ -19 \mathrm{a} & 179: 402 & 750 \pm 90 & \text { 1600 B.C. } \\ -19 \mathrm{~b} & 179: 402 & 950 \pm 100 & \text { A.D. } 1200 \\ -20 \mathrm{a} & 179: 401 & 730 \pm 90 & \text { A.D. } 1000 \\ -20 \mathrm{~b} & 179: 401 & 700 \pm 110 & \text { A.D. } 1220 \\ -21 & 179: 400 & 830 \pm 110 & \text { A.D. } 1250 \\ -22 & 179: 402 & 940 \pm 60 & \text { A.D. } 1120 \\ -23 & 179: 402 & 640 \pm 80 & \text { A.D. } 1010 \\ -24 & 179: 402 & 960 \pm 100 & \text { A.D. } 1310 \\ -25 & 179: 405 & 340 \pm 100 & \text { A.D. } 990 \\ -26 & 179: 400 & 600 \pm 100 & \text { A.D. } 1610 \\ -27 & 179: 406 & 1090 \pm 130 & \text { A.D. } 1350 \\ -28 & 179: 402 & 730 \pm 120 & \text { A.D. } 860 \\ & & & \text { A.D. } 1220\end{array}$




\section{Radiocarbon Measurements :}

$\begin{array}{lcc}-29 & 179: 406 & 2210 \pm 160 \\ -30 & 179: 403 & 740 \pm 90 \\ -31 \mathrm{a} & 179: 402 & 640 \pm 90 \\ -31 \mathrm{~b} & 179: 402 & 850 \pm 100 \\ -32 & 179: 402 & 720 \pm 100 \\ -33 & 179: 400 & 760 \pm 100 \\ -34 \mathrm{a} & 179: 401 & 370 \pm 100 \\ -34 \mathrm{~b}^{3} & 179: 401 & 500 \pm 80 \\ -36 & 179: 402 & 660 \pm 100 \\ -37 & 179: 405 & 1590 \pm 80 \\ -38 & 179: 407 & 2720 \pm 90 \\ -39 & 179: 402 & 980 \pm 80 \\ -40 & 179: 403 & 370 \pm 60 \\ -41 & 179: 403 & 3750 \pm 100 \\ -42 & 179: 403 & 760 \pm 90 \\ -43 & 179: 403 & 560 \pm 80 \\ -44 & 179: 404 & 730 \pm 80 \\ -46 & 179: 404 & 1050 \pm 60 \\ -48 & 179: 407 & 12,800 \pm 220 \\ -49 & 179: 404 & 1070 \pm 80\end{array}$

260 B.C.

A.D. 1260

A.D. 1310

A.D. 1100

A.D. 1230

A.D. 1190

A.D. 1580

A.D. 1400

A.D. 1290

A.D. 360

870 B.C.

A.D. 970

A.D. 1580

1800 B.C.

A.D. 1190

A.D. 1390

A.D. 1220

A.D. 900

10,850 B.C.

A.D. 880

1 M-507.

${ }^{2} \mathrm{M}-468$

${ }^{3}$ Considered more reliable than date of $34 a$.

\section{Yale University Radiocarbon Laboratory ${ }^{1}$}

\begin{tabular}{|c|c|c|c|c|c|c|}
\hline $\begin{array}{l}\text { Laboratory } \\
\text { number }\end{array}$ & $\begin{array}{c}\text { Published } \\
\text { reference }\end{array}$ & $\begin{array}{l}\text { Original date or } \\
\text { other value }\end{array}$ & $\begin{array}{l}\text { Corrected } \\
\text { or other } 2\end{array}$ & $\begin{array}{l}\text { date } \\
\text { alue }\end{array}$ & $\partial \mathrm{C}^{14}, \%$ & $\begin{array}{c}\text { A.D./ } \\
\text { B.C. date }\end{array}$ \\
\hline Y.4 & $3: 955$ & $1540 \pm 130$ & & & & \\
\hline-5 & $3: 955$ & Modern & & & & \\
\hline-6 & $3: 955$ & $1520 \pm 140$ & & & & \\
\hline-7 & $3: 955$ & $760 \pm 130$ & & & & \\
\hline-8 & $3: 955$ & $4040 \pm 150$ & & & & \\
\hline-9 & $3: 955$ & $4680 \pm 160$ & & & & \\
\hline .10 & $3: 955$ & $7050 \pm 240$ & & & & \\
\hline-11 & $3: 957$ & $2830 \pm 130$ & & & & \\
\hline-13 & $3: 957$ & Moderns & see Y.11 & $d$ & & \\
\hline .19 & $3: 959$ & $5870 \pm 100$ & & & & \\
\hline-20 & $1: 287$ & $5.44 \pm 0.11 \mathrm{cpm}^{2}$ & & & $-90 \pm 20 \mathrm{a}$ & \\
\hline-21 & $1: 287$ & $6.00 \pm 0.10 \mathrm{cpm}^{2}$ & & & $+3 \pm 17 \mathrm{a}$ & \\
\hline .22 & $1: 287$ & $6.11 \pm 0.08 \mathrm{cpm}^{8}$ & & & $\begin{array}{r}+22 \pm 13 a \\
\text { a }\end{array}$ & \\
\hline-27 & $1: 287$ & $5.35 \pm 0.09 \mathrm{cpm}^{2}$ & & & $-105 \pm 17$ a & \\
\hline-29 & $1: 287$ & $5.99 \pm 0.12 \mathrm{cpm}^{8}$ & & & $0 \pm 20 \mathrm{a}$ & \\
\hline-30 & $1: 287$ & $6.20 \pm 0.08 \mathrm{cpm}$ & & & $+37 \pm 13$ a & \\
\hline-32 & $3: 955$ & Modern & & & & \\
\hline-36 & $4: 909$ & Modern & & & & \\
\hline-37 & $3: 955$ & Modern & & & & \\
\hline-38 & $3: 959$ & Modern & & $d$ & & \\
\hline-40 & $3: 959$ & $2850 \pm 120$ & & $\mathrm{~d}$ & & \\
\hline-41 & $3: 959$ & $6250 \pm 380$ & & $d$ & & \\
\hline-42 & $3: 959$ & $2870 \pm 130$ & & & & \\
\hline .43 & 3:959 & $2700 \pm 130$ & & & & \\
\hline$-4,4$ & $3: 959$ & $2570 \pm 130$ & & & & \\
\hline-49 & $4: 918$ & $>38,000$ & & & & \\
\hline .56 & $1: 287$ & $5.93 \pm 0.12 \mathrm{cpm}^{8}$ & & & $-8 \pm 20 \mathrm{a}$ & \\
\hline-63 & $1: 287$ & $6.03 \pm 0.10 \mathrm{cpm}^{8}$ & & & $+8 \pm 17$ a & \\
\hline-64 & $3: 957$ & $2560 \pm 200^{\mathrm{s}}$ & see Y-11 & $\mathrm{d}$ & & \\
\hline-65 & $3: 957$ & Modern ${ }^{\mathrm{s}}$ & see $\mathrm{Y}-11$ & $\mathrm{~d}$ & & \\
\hline .72 & $3: 960$ & Modern & & & & \\
\hline-85 & $3: 960$ & $2720 \pm 130$ & & & & \\
\hline $.86^{2}$ & $3: 960$ & $4100 \pm 450^{\mathrm{s}}$ & & $d$ & & \\
\hline
\end{tabular}

